\title{
Werner Abraham
}

\section{Faszination der kontrastiven Linguistik ,DaF': der Para- meter, schwere/leichte' Sprache unter typologischer Sicht}

\section{Einleitung: worum es geht}

Wenn man mit linguistisch gewieften Amerikanern spricht und ,zur Sache kommt' (d.h. keine bloß höflichkeitsbedingte Zurückhaltung mehr einzurechnen hat), dann hört man oft folgendes: das Englische sei zurecht die Lingua franca der Diskurse der modernen wirtschaftlichen, technischen, kulturellen und akademischen Welt; denn welche andere Sprache sei direkter, sachlicher - und: einfacher, internationaler', leichter, sicherlich im Vergleich zum Deutschen mit seiner exotischen Wortstellung, über die sich Mark Twain - dem Sinne nach nicht nur einmal - so äußerte: ,Wenn ein Deutscher mit einem Satz im Munde an der deutschen Atlantikküste in die See taucht und nach Wochen an der amerikanischen Ostküste wieder auftaucht, dann hat er, wenn man Glück hat, gerade das Verb des abhängigen Satzes im Munde.' - so über die unmögliche Wortstellung des Deutschen und das unendlich lange, nie aufhören wollende Mittelfeld - und weiter, wieso denn um Gottes willen Mädchen oder Knäblein sächliches Geschlecht haben könnten und was der Deutsche damit genau meine, wo beide doch bereits eindeutige geschlechtliche Merkmale aufwiesen - somit die Unnötigkeit, ja die Absurdität des grammatischen nominalen Genus im Deutschen anprangernd.

Man braucht, um gleich zur Sache der Wissenschaft zu kommen, nicht die Junggrammatiker Schmidt und Schleicher als Advokaten der akademischen Position zu den Unterschieden zwischen, weiter entwickelten ' und ,weniger weit entwickelten' Sprachen der Welt zu bemühen. Hermann Paul (1880) hat sich erst gar nicht mit solchen pseudodarwinschen Evolutionswertungen aufgehalten. Nach Paul spielen Vereinfachung und Komplizierung in der Sprachevolution komplementäre Rollen. Ich möchte dieses Paulsche Diktum im folgenden veranschaulichen und anhand des durch die formal-typologische Sicht der modernen Linguistik erstellbaren Aussagewerts deskriptiv griffi-

1 „The problem with defending the purity of the English language is that English is about as pure as a cribhouse whore. We don't just borrow words; on occasion, English has pursued other languages down alleyways to beat them unconscious and rifle their pockets for new vocabulary." - James D. Nicoll 
ger machen und grammatiktheoretisch unterbauen. Und komme gleich zur Sache: Es soll gelten vor diesem angekündigten Hintergrund zwei Argumentationslinien zu verfolgen: zum einen die Ausbildung des modernen Englischen aus dem dem modernen Deutschen so strukturähnlichen Altenglischen, in dessen Ausbildungsverlauf Vereinfachung über Komplizierung in einem Maße dominierte, das sich in keiner anderen germanischen Sprache wieder findet - der kontrollierende Faktor dabei war ein soziohistorischer, der die Weitergabe der Grammatik über die Generationen hinweg überlagerte und deren grammatisch-diachrone Autonomie unterbrach; und zum anderen will ich einige außergrammatische Prozesse und Faktoren ansprechen, die zur Vereinfachung und Komplizierung mit überlagertem, modulwechselndem Vorteilsgewinn beitragen. Damit gehen wir weit über jene junggrammatischen ästhetischen Urteile hinaus, die wir alle ganz gerne nachbeanspruchen würden, aber uns aus politikgerechter Perspektive nicht so recht trauen. Mit einem Wort: da ist was dran an der Schleicherschen Evolutionsthese, aber es ist eben doch nur sehr oberflächlich was dran, das bei tieferer Sicht ganz anders dargestellt werden muss.

Der Grundgedanke der folgenden Ausführungen ist der: das Englische als aktuelle Lingua franca der Welt - verzichtet auf ,Schnörksel ${ }^{*}$ - auf Überspezifikation und Komplikationen -, die dem Deutschen immer eigen waren und heute noch immer eignen - die es nicht nur nicht abzulegen gewillt ist, sondern die es gar nicht ablegen kann, da gerade diese, schweren ' Eigenschaften des Deutschen unter einer bestimmten Perspektive zu einer leichteren Sprache machen. Ich will zuerst den Gesichtspunkt der Überspezifikation des Deutschen im Vergleich mit anderen germanischen Sprachen illustrieren und in einem zweiten Abschnitt auf jene Gesichtspunkte eingehen, unter denen derartige formale ,Redundanzen' entscheidende Vorteile erbringen.

\section{Das Wesen von Über- bzw. Unterspezifikation und Vereinfachung}

Folgen wir zuerst der Geschichte des Englischen, einer Sprache, der man ja eine starke Eignung als globale Lingua franca vor allem deshalb nicht absprechen kann, weil sie dem Lerner so viel weniger formale Hürden als andere europäische Sprachen stellt. Wenn man den Sprachhistorikern und Soziolinguisten folgt, dann hatte im Mittelenglischen bereits starke autonome Formenvereinfachung bzw. Teilkreolisierung ${ }^{2}$ unter Einfluss des Romanischen der Normannen nach Wilhelm dem Eroberer stattgefunden. Die Diskussion allerdings, wieso Kreolisierung eingesetzt hatte $-d$.h. welche Kre-

${ }^{2}$ Thomason/Kaufman (1988) und McWhorter (2001) stehen auf dem Standpunkt, dass nicht von Kreolisierung gesprochen werden kann, da bereits den skandinavischen Dialekten, die letztlich die alten angelsächsischen Dialekte überlagerten, die Überspezifikation fehlte. Es entstand ,Unterspezifikation' demnach nicht erst durch die sprachliche Mischung. 
olisierungskriterien gerade fürs Englische haftbar gemacht werden müssen -, diese Frage ist nicht erledigt. Schließen wir uns einer Minimalposition an: der Verlust von Flexion, wohl nur die Spitze des Eisbergs an Veränderungen im Englischen, geht auf ein Abgleichen von Überspezifikation jener formalparadigmatischen und intensional-semantischen Merkmale (Flexions- und Derivationsmorphologie) zurück, die nur im Deutschen erhalten geblieben sind, die allerdings in den anderen germanischen Sprachen ebenfalls zum Großteil beseitigt wurden, wenn auch in etwas geringerem Umfang als im Englischen. Gleichzeitig - oder besser: parallel mit diesem Abglich formalparadigmatischer Unterscheidungen - trat auch ein Abglich syntaktischer Komplexität ein. All dies resultiert in einer verhältnismäßig isolierten Stellung des Englischen gegenüber den anderen germanischen Sprachen, insbesondere den kontinental-westgermanischen Vertretern - dem Deutschen, Niederländischen und Westfriesischen. Teil der hier eingenommenen methodischen Minimalposition ist auch, dass wir davon ausgehen, dass dieser Abschliff von morphosyntaktischen und semantischen Unterscheidungen durch die jahrhundertelangen Kontakte von Normannen und Anglo-Sachsen begünstigt wurde, aber dass Vereinfachung der Komplexität bereits weit fortgeschritten war, als die französischsprechenden Normannen in England eindrangen. Schließlich haben alle heutigen skandinavischen Sprachen ihren morphologisch-syntaktischen Bestand durchaus ähnlich abgebaut wie das Englische: damit auch OV, damit auch die strenge V2-Stellung des Deutschen, Ndl. und Westfriesischen, die strenge Letztstellung der starkbetonten Verbpartikeln, der Abbau der morphologisch gestützten Aspektparadigmatik, vor allem aber - einhergehend mit der Einschränkung des deutschen syntaktischen Mittelfelds - den für die Diskursprominenz des Deutschen so wesentlichen freien Umstellungsprozess (Scrambling), der nur mehr dem Deutschen (und mit Einschränkungen dem Ndl.) eignet.

Es wird sich zeigen, dass das Englische kaum weniger überspezifiziert als seine nordgermanischen Verwandten - jedenfalls den festlandskandinavischen Sprachen - im Gegensatz jedenfalls zu den westgermanischen ,Brüdern' auf dem Festland. Dieser Vergleich liefert ein nicht unwichtiges Nebenprodukt der vorliegenden Diskussion, nämlich dass das Englische erst durch Mischung mit dem Romanischen seiner normannischen Eroberer d. h. durch Kreolisierung (vgl. Fn. 2) - sich zu jener morphologiearmen und syntaktisch entkomplizierten Sprache ausgebildet hat, die es heute darstellt (so schon Thomason/Kaufman 1988; McWhorter 2001).

Ein kurzes methodisches Caveat sei an dieser Stelle eingefügt. Überspezifikation im Unterschied zu Unterspezifikation sollte deutlich und nachvollziehbar sein vor dem Hintergrund des Vergleiches der Flexions- und Ableitungsparadigmen im Englischen und im Deutschen und sowie den anderen germanischen Sprachen. Komplizierung bzw. Vereinfachung dagegen bedürfen einer eigenen Rechtfertigung. Kurz gesagt liefert erst eine regelverknüpfende und regelrechtfertigende Grammatiktheorie wie die generative Mittel, 
diesen Begriff einleuchtend zu verwenden: eine sprachliche Struktur sei komplizierter, wenn sie mehr Ableitungsoperationen - oder, in einem rein repräsentativen Modell wie HPSG oder Bresnans LF-Theorie, mehr unabhängige, $d . h$. nicht voneinander ableitbare Termeinträge - verlangt als die sprachliche Struktur einer anderen Sprache. Das einfachste Beispiel sind Sprachen mit gespaltener Ergativität (etwa Hindi, Urdu, Balutschi), wo das Nominativ-Akkusativ-Kasussystem im Präsens und Imperfektiv, das Ergativ-Absolutiv-Kasussystem dagegen im Perfekt und Perfektiv eingesetzt wird. Noch ein einfacheres Beispiel liefert eine Sprache, die nicht in jedem Fall der Satzableitung das Verb nach $C^{\circ}$ (Comp; siehe Tabelle 6 unten) versetzen muss, sondern es auch bereits in $\mathrm{I}^{\circ}$ (Inflection $=$ Verbflexionskategorie im unabhängigen Satz) stehen lassen kann (wie etwa in den thetischen Sätzen des Deutschen). Ich werde genau von diesem grammatischtheoretisch-methodischen Begriff der Strukturvereinfachung Gebrauch machen bei der Begründung im letzten Abschnitt, warum das Deutsche trotz - nein: gerade infolge - aller formal-morphologischen Überspezifikationen bzw. Redundanzen eine wesentlich diskursvereinfachende Sprache (geblieben $\left.{ }^{3}\right)$ ist.

\section{Beispiele zur typologischen Einordnung und zur Sonderstellung des Deutschen innerhalb der Germania}

\subsection{Sekundäre Typologiemerkmale des Deutschen: der Parameter der Referenzwiederaufnahme}

Es sollen im Folgenden zwei Parameter der hörsprachlichen Erleichterung, also der Verarbeitungs- (Parsing-)erleichterung und Texteinbettung unterschieden werden: jener der Erleichterung der Referenzwiederaufnahme sowie jener des schnelleren und eindeutigen Textanschlusses. Beide Kriterien sind beim oralen Sprachkode von primärer Bedeutung; letzteres Kriterium allerdings, das des schnelleren und eindeutigen Textanschlusses verleiht auch dem geschriebenen Kode eine eigene Qualität. Ich komme auf Kriterien des Textanschlusses eigens zurück, gehe aber zuerst auf die Kriterien der Referenzwiederaufnahme ein. Unter Referenzwiederaufnahme versteht man alle Kodierungen, die anaphorische Verweise aufgrund von Numerus, Person, Geschlecht, thematischem Status u.e. a. erleichtern bzw. eindeutig machen. Zum Beispiel kann das Deutsche auf Mädchen oder Weib mit es bzw. sie zurückverweisen, je nachdem womit man die Referenz neben anderen Anaphern eindeutiger herstellen kann. ${ }^{4}$

3 Soweit wir wissen, verfügte das Indogermanische über V1, V2 im Kompensat mit V-letzt (somit Verbklammer) sowie unabhängiges V-letzt im Deklarativ; Negationsklitika und Negationshäufung; reiche morphologische Vernetzung; keine Unterscheidung definiter und indefiniter Artikel; Modalpartikel; sowie Aspektund Aktionsartmarkierung.

${ }^{4}$ Damit lässt sich das Argument Mark Twains, die grammatische Genuszuordnung im deutschen sei willkürlich und unnatürlich, trefflich entkräften. 


\subsubsection{Markierung inhärenter Reflexivität}

In der Germania herrscht Uneinigkeit hinsichtlich der Markierung dessen, was im Deutschen, (un)echte Reflexivität', in der englischen Literatur (unglückseligerweise) manchmal auch ,inhärente Reflexivität" heißt (Haiman 1983, Klaiman 1991, Kemmer 1993). Inhärente Reflexiva sind durch Grammatikalisierung und anschließende semantische Ausbleichung (Deintensionalisierung) des Reflexivpronomens entstanden (man denke an das Reflexivpassiv im Skandinavischen) und zwar bei Verben, die, inhärent-reflexive Tätigkeiten denotieren, vor allem bei Bewegungsverben. Im Deutschen gehören dazu sich beeilen - nicht jedoch sich erinnern (da man ja andere Akkusativobjekte einsetzen kann), wohl wieder sich benehmen ,to behave'). Glaubt man Kemmer (1993) oder Peitsara (1997), dann ist dies eine allgemeine Entwicklungstendenz in allen Sprachen.

Die Markierung solch inhärenter Reflexivität ist allen germanischen Sprachen eigen mit Ausnahme des Englischen. Hier hat das Ne. am radikalsten vereinfacht - mit dem Ergebnis, dass oft nur aus dem Kontext ableitbar ist, ob das Verb transitiv oder (reflexiv) intransitiv verwendet ist (vgl. Abraham 1997a)

Tabelle 1: Inhärente Reflexiva im Germanischen (nur in der 3. Pers.)

\begin{tabular}{|l|c|c|c|c|c|c|c|c|c|c|c|}
\hline & Dt. & Ndl. & Fr. & Afrik. & Fldskand. & Isl. & Fär. & Jidd. & Obdt. & Ae. & Ne. \\
\hline $\begin{array}{l}\text { explizites } \\
\text { Reflexiv- } \\
\text { pronomen }\end{array}$ & + & $(+)$ & & & + & + & + & + & $(+)$ & & \\
\hline $\begin{array}{l}\text { Personal- } \\
\text { pronomen }\end{array}$ & & & + & + & & & & & $(+)$ & + & \\
\hline $\begin{array}{l}\text { keine } \\
\text { Anzeige }\end{array}$ & & $(+)$ & & & & & & & & & + \\
\hline
\end{tabular}

[LR= linksläufige Rektion; $R R=$ rechtsläufige Rektion ]

(1) Beispiele zur typologischen Verteilung der Reflexivrektion:

Dt.

Ndl.

Fris.

Afrik.

Fldskand. (Schw.) RR Isl.
LR sich rasieren, sich beeilen, sich erinnern

LR (zich) scheren, (zich) bewegen, (zich) herinneren

LR hy skeart him, hy skamje him, hy stel him foar hy bevind hom, hy roer hom, hy herinner hom raka sig, röra sig, känna sig komast „erreichen, ankommen“, snúa sér „,sich umdrehen", skammast sin „,sich schämen“" 
Fär.

RR aka scer „sich rasieren“, snúgva scer „sich umdrehen“, atla sær "sich vornehmen"

Jidd.

$\mathrm{RR}\left(\mathrm{LR}^{5}\right)$ bukn zich, shlaychn zich, schemen zich

Wenn hier zusätzlich etwas auffällt, dann ist dies die Rektionsrichtung: nur Ndl., W-Fries. sowie Dt. regieren nach links; alle anderen Sprachen haben rechtsläufige Rektion. Die Reflexivanzeige fehlt im gesamten Ingwäonischen, so Friesischen, z. T. (allerdings erst nach neueren Entwicklungen) im Ndl. - völlig dagegen im Afrikaans. Zudem beschränkt sich die kategorienautonome Reflexivanzeige auf die 3. Person - in den anderen Personen wird durchwegs das Personalpronomenparadigma herangezogen. Zu betonen ist, dass das Ne. wohl eine neue Reflexivanzeige zur Verfügung hat (my-, your-, him/herself), aber nur zur Kontrasthervorhebung bzw. für die Anzeige des syntaktischen Reflexivs (,Bindung') verwendet. Eigentlich dürfte diese aus dem Intensivpronomen abgeleitete neue Reflexivform gar nicht zum etymologischen Reflexiv gerechnet werden.

Abschließend lässt sich also sagen, dass „English became unique among Germanic languages in an individual tendency to treat overt reflexivity as redundant, unless marked for practical or stylistic reasons" (Peitsara 1997, S. 337), dass alle anderen germanischen Sprachen - vor allem das Festlandskandinavische - jedoch weitaus sparsamer in der Reflexivanzeige sind als das Deutsche. Wie Curme (1952, S. 331) hervorhebt, „German is usually tenacious of reflexive form even after its meaning has changed“. Das Deutsche schließt sich mit der Verwendung des Reflexivs zur Diatheseanzeige dem Skandinavischen an. In der Ableitung des Mittelverbs (vgl. das antikausative die Tür öffnet sich; vgl. schwed. minna-s ,,sich erinnern") ebenso wie der Mittelkonstruktion (diese Brötchen verkaufen sich rasend) stellt es sich zum skandinavischen $s$-Passiv (dem ,Reflexivpassiv, dem ja ein analytisches bliva-Passiv mit anderen Funktionen zur Seite steht). Haspelmath (1998, S. 276) meint darin ein paneuropäisches Sprachbundmerkmal erkennen zu müssen. Es ist jedoch gezeigt worden, dass die einzelnen Sprachen mit dem Reflexiv als Diatheseanzeige ebenso unterschiedlich umgehen wie beim lexikalischen Reflexiv. So haben das Ndl. und W-Fries. überhaupt keine Diatheseanzeige bei der Antikausativkonstruktion (vgl. deze schoenen lopen heerlijk zachtjes, diese-Schuhe-laufen-herrlich weich ${ }^{6}=$, in diesen Schuhen läuft es *(sich) herrlich weich"), ebenso wenig wie das Englische (these bagels sell quickly), aber eben im Unterschied zum Skandinavischen.

Ganz klar sind die Folgen dieser fehlenden Reflexivanzeige im gesamten ,Ingwäonischen" (dem Westgermanischen mit Ausnahme des Deutschen): lexikalische Transitivität lässt sich von Intransitivität eines Verbs formal nicht mehr unterscheiden, damit auch nicht, ob ein solches bi-transitives

${ }^{5}$ rechtsläufige Rektion, allerdings mit prinzipiell möglicher V-Anhebung über das direkt Objekt hinweg (Anhebung natürlich nach links), so dass das IO oft in sichtbar linksläufiger Rektion steht; vgl. Santorini (1993). Siehe (11a) weiter unten. 
Verb transitiv-elliptisch oder intransitiv verwendet wird (Abraham 1997a). Damit geht einher, dass inhärent-intransitive Verben tendenziell auch transitiv verwendet werden (vgl. ne. walk the dog). Vissers diagnostizierte einen allgemeinen „drift towards transitivization“ im Englischen (Visser 1963, S. 127-35). In den deutschen Mundarten dagegen hat sich die gegenläufige Tendenz durchgesetzt (was ja beweist, dass Konservativität nicht (nur?) Sache unserer Grammatikschreiber ist): man denke an den Schwund des ae. noch vorhandenen Perfektivpräfixes ge- (ae. aernan „laufen“, gecernan ,erreichen"), das oberdeutsch ersetzt ist durch das Aktionsartableitungspräfix (d)er-: (sich) derrennen/derstessen ,sich zu Tode rennen/stoßen“). Dass in solche Zusammenhänge auch der Schwund des Umlauts als Diatheseanzeige gehört, sei hier nur angedeutet (im Deutschen erhalten, - vgl. sinken und senken, ne. nur sink). Das Deutsche spezifiziert ausnahmslos; alle anderen Sprachen verstehen sich zu sparsameren Mitteln oder zum gänzlichen Verzicht dieses lexikalischen und syntaktischen Mittels.

Das Altnordische ließ die klitischen Reflexiva der 1. und 3. Person noch als Suffix erkennen: bindomk, ,ich binde mich", bysk ,sich wappnen“, staksk "sich fortstoßen" (Heusler 1950, S. 107). Diese Strategie wurde auf die inhärente Reflexivierung erweitert: their setiask nithr ,sie setzten sich nieder", er hefnezk á honom ,du rächst dich an ihm“, thetta felsk honom vel i skap „das fühlt sich gut an; das passte ihm gut" (Heusler 1950, S. 137-8). Aber auch das freie Reflexivpronomen war möglich, wenn auch weitgehend auf den Dativ eingeschränkt: hann brá sér ,er erging sich“, das allerdings auch wegbleiben durfte (hann brásk) (Heusler 1950, S. 138).

\subsubsection{Anzeige des externen Besitzers}

Schon beim dem Dt. so nahen Ndl. fällt auf - und ist Gegenstand der besonderen DaF-Lehre -, dass es keinen possessiven Dativ gibt wie in

(2) Beispiele zur typologischen Verteilung des Besitzausdrucks:

Dt. Die Mutter wäscht ${ }_{3}\left[_{\text {Posst:sso o }}\right.$ dem Kind $] \int_{\text {POSSEDE }}$ die Haare $]=$

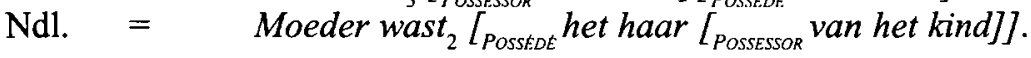

Dt. Er bricht $t_{3}$ sich das/sein Bein

Ndl. = Hij breekt ${ }_{2}\left({ }^{*}\right.$ zich) het $/ z$ 'n been

Im Dt. gilt dies als Dativus sympathicus, international-linguistisch hat sich der Terminus external possessor (marking) durchgesetzt (König/Haspelmath 1998). Die Eigenheit des Deutschen ist, dass der Possessor als eigene Konstituente konstruiert wird und nicht nur als Subkonstituente der possédé-NP (die Haare) wie im Ndl. Es folgt auch, dass das Verb im Deutschen dreiwertig ist, im Ndl. dagegen zweiwertig. 


\begin{tabular}{|l|c|c|c|c|c|c|c|c|c|c|c|}
\hline & Dt & Ndl & Fr & Afrik & Skand & Isl & Fär & Jidd & Obdt & Ae & Ne \\
\hline $\begin{array}{l}\text { Besitzer- } \\
\text { dativ }\end{array}$ & + & $(+)$ & $(+)$ & & & & & + & + & & \\
\hline $\begin{array}{l}\text { Besitzer- } \\
\text { lokativ }\end{array}$ & & & & + & + & + & + & & & & + \\
\hline $\begin{array}{l}\text { Besitzer-NP } \\
\text { sub Besitz-NP }\end{array}$ & & $(+)$ & $(+)$ & & & & & + & + & + & \\
\hline
\end{tabular}

Tabelle 2: Anzeige des externen Besitzers

Bereits das Altenglische kannte die externe Possessoranzeige, die freilich im Ne. völlig verloren gegangen ist:

(3) Beispiele zur typologischen Verteilung des Besitzausdrucks:

Ae. $\quad$ tha cnitton hi rapas ... hire to handum and fotum (Mitchell 1985, S. 125) ,dann binden sie (das) Seil ihr an Hände und Füße'

Dt. $\quad$ sich das/sein Bein brechen (*sein Bein brechen), dem Kind die Haare waschen

Ndl. (hem) zijn arm breken

Fris. $\quad$ my de/myn holle stompen „mir/meinen Kopf stoßen“

Schwed. nagon bröt armen på honom, jem. brach den Arm an ihm"

Isl. han nuddadi á henni fatur-na ,er massierte an ihr die Beine“

Fär. $\quad$ eg hoyrdi røddina á honum , ich hörte (die) Stimme an ihm

Jidd. a di mame hot em gewascht di hor, die Mutter hat ihm gewaschen die Haare'

b di mame hot gewascht di hor farn kind, die Mutter hat gewaschen die Haare vom Kind"

Jiddisch-b ist typologisch keine eigenständige Alternative; sie kommt in jedem der deutschen Dialekte ebenfalls vor.

Die Konstruktion des externen Besitzes tritt in allen germanischen Sprachen außer dem Englischen und dem Afrikaans auf. Sie bezieht sich im allgemeinen auf belebte Besitzer, also auf Körperteile, Verwandte, Kleidung, Behausungen und weniger oft auch auf Gefühlsbewegungen, ganz allgemein somit auf Teil einer „persönlichen Sphäre“ (König/Haspelmath 1997, S. 530-33). Allerdings zeigt sich der deutsche Dativpossessor im Festlandskandinavischen und Isländischen als Lokativ, im Jiddischen als Dativ bei Pronomina und als Lokativ bei Appellativen. Ne. tritt ein Personssympathicus kaum mehr auf: vgl. (4a) und (4b).

(4) a She looked him in the eyes

b She died on me 
Der Verlust in allen Sprachen außer dem Deutschen kann nichts mit dem Verlust des Kasusunterschieds zwischen dem Dativ und Akkusativ zu tun haben, denn dann müssten im Ndl. noch deutlichere Spuren davon existieren. Selbst dem im Isländischen vagierenden Grammatikbegriff der ,Dativtendenz $z^{6}$ d. h. der Bindung zwischen Experiencervalenzen und dem Dativ setzt das Deutsche seine unerschütterliche Kasuskonservativität entgegen

(5) a mig brestur kjark mich gebricht Mut.ACC

b mér brestur kjarkur mir gebricht Mut. NOM „Mir gebricht es an Mut"

(6) mich schwindelt/grau(s)t/ödet an

Was zu beachten ist und dem Deutschen eine Sonderrolle in der Germania einräumt, sind allerdings nominale Possessivkonstruktionen wie in (7).

(7) a dem Vater(/dem/ihm) seine Zigarre, der(/*ihr) ihre Schürze

Das Auffällige ist dabei, dass die nominal selbständige Konstruktion jene in der verbalen Rektion - vgl. Dt. unter (7) oben - direkt kopiert:

(8) sich (=Dativ!) (das/)sein Bein (brechen), dem Kind die Haare (waschen)

Das gibt es sonst nur im Ndl., allerdings dort ohne eigene Dativanzeige. Abgesehen davon steht das Deutsche somit mit der Anzeige der EXP-Rolle in der Germania nicht alleine da. Nur das Englische und das Afrikaans nehmen sich dabei völlig aus. Englische Homonymien von semantisch Verschiedenem wie in He grabbed my hand und He grabbed my folder vermeidet jedoch nur das Deutsche kasusaufwendig und anders als jede andere germanische Sprache. Dies ist, so wollen wir sagen, überspezifizierend.

Wie sonst auch setzt das Skandinavische statt eines Dativs einen Präpositionslokativ (Abraham 2000).

Norwegisch:

(9) a Någon bröt armen på honom.

jemand brach.Praet Arm.DEF an.PräP ihm.Pers.Pron.3SG. „Jemand brach ihm den Arm.“ (König/Haspelmath 1997, S. 559)

Isländisch:

b Han nuddaDi á henni fætur-na.

er massierte an ihr Bein.PL-DEF:ACC

„Er massierte ihr die Beine.“ (König/Haspelmath 1997, S. 559)

Färöisch:

c Eg hoyrdi røddina á honum.

Ich hörte Stimme an ihm

„Ich hörte seine Stimme.“ (Lockwood 1955, S. 105) 
Ein solcher Analytisierungsprozess ist für Sprech- und Koinesprachen nicht unselten (vgl. den Ersatz des einfachen Präteritums durch die Perfektperiphrase im Afrikaans, Jiddischen und (gesprochenen) Oberdeutschen; Abraham/Conradie 2001).

\subsubsection{Grammatisches Geschlecht angezeigt am Artikelwort}

Unter den germanischen Sprachen ist das Deutsche die einzige Sprache mit der relativ eindeutigen Genusdreiteilung ,maskulin-feminin-neutrum', dies noch dazu als grammatisch-morphologischer Klassifikationsgesichtspunkt und keinesfalls als Sexuskriterium. Am anderen Ende der Unterscheidungsskala steht das Englische, das seit dem Ende des 12. Jh.s - übrigens vom Norden nach Süden ausfächernd - keinerlei Genusunterscheidung mehr formalisiert. Dies ist überraschend deshalb, weil alle Schwestersprachen des Englischen durchaus ähnlichen phonetischen Erosionen unterlagen, keinesfalls jedoch den Einheitsartikel mit dem Neuenglischen teilen. Zudem haben alle germanischen Sprachen die germanische Erstsilbenbetonung gemein, die die Erosion der Flexions- und Ableitungsmorphem begünstigte. Das Deutsche gab dem nicht nach, im Gegensatz zu seinen Schwestersprachen. Das Ae. verfügte wie das moderne Deutsche über ein reiches Genusklassifikationssystem, in das sich die Artikelwörter einreihten: se (masc.), seo (fem.) and thoet (neut.) - all dies kollabierte im Ne. zum genusneutralen the. Zur Unterscheidungslosigkeit des Englischen stellt sich bloß das Afrikaans - welches in seiner Entfaltung ja heftigstem Druck von Zweitspracherwerb und Kreolisierung ausgesetzt war. Vergleichbar sind in den europäischen Sprachen ausschließlich Dialektvarietäten wie das Westdänische (Haberland 1994), das ostrobothnisch-schwedische Tamian Lettische (Mathews 1956) sowie Mandresalbanisch (Hamp 1965), wo zudem noch Sprachkontakte (Livonisch, Türkisch) zur totalen Genusaufgabe führten.

Ist Genusmarkierung im Deutschen völlig nutzlos, totaler Luxus? Natürlich nicht: die Referenzwiederaufnahme etwa in der Form von Pronomina ist über Genusspezifikation viel leichter; umständlichere Syntaxspezifikationen sind nicht nötig. Es ist wohl mit Trudgill (1999) davon auszugehen, dass sich grammatische Genusunterscheidungen im Urindogermanischen ausbildeten, um Referenzeindeutigkeit im fortlaufenden Text zu fördern. Hat hier Exaptation im Sinne von Kellers unteleologischem Sprachwandel über die ,unsichtbare Hand' stattgefunden, derart dass die Klassifikationsmerkmale schließlich zu Genusunterscheidungen zweckentfremdet wurden? Ist das vielleicht sogar der generelle Erklärhintergrund zu vielen Erscheinungen, die ausschließlich das Deutsche auszeichnen? Was Zweckhaftigkeit betrifft, so muss man angesichts der anderen germanischen Sprachen, die ja ohne die Genusunterscheidung des Deutschen funktionieren, sicher von Überspezifikation sprechen.

$\mathrm{Zu}$ vermerken ist an dieser Stelle nur, dass die Textreferenzerleichterung durch Genusmorphologie zu jenen Vorteilen gehört, die ich für das Deutsche annehme. Doch dazu später. 


\subsubsection{Wortableitungsmorphologie}

Das Deutsche hat nicht nur unerhörten Reichtum an Ableitungsaffixen und -affixoiden; es baut sein Inventar vor allem über Affixoide dauernd produktiv aus, und kein Wörterbuch ist imstande, diesen Neubildungen zu folgen oder sie vorauszusagen. Am anderen Ende der Bildungsskala steht wieder das Englische, das so gut wie keine Affixe erhalten hat - wiewohl es, wie die Affix- und Partikelneubildungen zeigen (etwa anhand der Sekundärprädikationen), dazu durchaus eine Verwendungslücke verspürt. Gehen wir dem in den beiden folgenden Kapiteln dazu etwas im einzelnen nach.

\subsubsection{Unterschiedlicher Spezifikationsabbau: die ,syntaktisch trennbare Verbpartikel und Verbpräfigierung}

Deutlichste Spur des ursprünglichen OV-Stellungs- und Rektionstypus des Englischen ist der Umstand, dass Verbpartikel ebenso postverbal stehen wie im Deutschen und somit eine Spur der ursprünglichen Verbklammer (SVOV) verraten. ${ }^{6}$

(10) a He put them uplover

$b$ He lead his guests over to the other bank of the river.

c *He put up/over them

$\mathrm{d}^{*} \mathrm{He}$ lead over his guests to the other bank of the river.

Wäre das Ne. ursprünglich SVO gewesen, dann sollte es festere Verbfügungen wie in (10e) geben - die es aber nicht gibt. Während das Ne. solche Verbfügungen als Infinitive getrennt notiert, zeigte das Ae. dem heutigen Deutschen äquivalente Zusammensetzungen (Unterstreichung für Worthauptakzent, was ja kennzeichnend für Verbpartikel im Unterschied zu Verbpräfix ist).

(10) e ae. toberstan > ne. to break apart, ae. inladan $>$ ne. to bring in, ae. forbarnan $>$ ne. to burn up

Der beste Nachweis für syntaktische Trennbarkeit ist der Umstand, dass die präponierten Partikel sich durch freie Adjektive wie down statt nither-, around statt $y m b$-, up und out anstatt des intensivierenden for-ersetzen und nach syntaktischer Erfordernis in der Verbklammer freistellen ließen: etwa in forbarnan wie in (10) oben.

Im Gegensatz zum syntaktischen Partikeltypus hat das Ne. die lexikalischen, unbetonten Verbpräfixe aufgegeben, offensichtlich durch allgemeinen Morphologieverlust - sicherlich früher und durchgehender dort, wo hauptsächlich grammatische Bedeutung vorlag.

${ }^{6}$ Dies ist der klarste Nachweis dafür, dass auch das Englische nicht, wie bei Kayne (1994) angenommen, grundlegend vom SVO-Typus ist, sondern sich eben von SOV aus entwickelte und noch Charakteristika dieses Stellungs- und Rektionstypus bewahrt hat. Siehe Haider/Rosengren (1998, S. 45 f.). Dasselbe Argument ist (gegen Zwart 2000) fürs Niederländische zu führen. 
(11) a Aufgabe des transitivierenden be-: ae. seon „sehen“, ae. beseon „besehen, schauen"

b Aufgabe des perfektivierenden bzw. transitivierenden ge-: ae. arnan „rennen“, ae. gearnan ,erreichen“ (vgl. oberdeutsch derrennen), ae. winnan ,sich mühen“, gewinnan ,besiegen“.

c Aufgabe des intensivierenden for-: ae. helan - forhelan ,hehlen - verhehlen", ae. tholian - fortholian ,entbehren“.

Deutsch ebenso wie Ndl., Fries., Jiddisch und Afrikaans haben solche Verbpräfixe im allgemeinen erhalten, wenn auch mit starker Abnützung der grammatischen Funktion und lexikalischen Bedeutung. Isländisch und Färöisch haben ähnlich betonte Partikel (,phrasal verbs') erhalten, jedoch die unbetonten Präfixe so gut wie abgebaut, einschließlich der Entsprechungen zu be-, ge- und vor-. Das Englische kommt ohne solche Spezifikationen aus, während die anderen germanischen Sprachen und das Deutsche im besonderen diese Spezifikation jedoch erhalten haben. Was bei McWhorter (2001) allgemein als ,trend towards analyticity" klassifiziert wird, ist bei genauerer Sicht unterschiedlicher Abbau bei lexikalischer Flexion und morphosyntaktischer Derivation. Derivation (Wortableitung) ist immer geringerer Erosion als Flexion ausgesetzt, wohl aus gutem Grund, macht Flexion doch grammatische Beziehungen durchsichtig, wogegen Wortableitung neue lexikalische Bedeutung schafft. So durfte im Englischen ebenso wie im Afrikaans das Infinitivsuffix fallen. Vgl. (12) zum Afrikaans, wo ableitendes $b e$ - erhalten ist, das Infinitivsuffix jedoch geschwunden ist (Ponelis 1993, S. 556-7).

(12) be-: beslis „beschließen“, vgl. ndl. beslissen, ont-: ontken „leugnen“, vgl. ndl. ontkennen, ver-: verpletter ,zerhauen“, ndl. verpletteren, verafrikaans ,afrikanisieren“" (ndl. verafrikaansen).

Die Funktion der Verbpartikel ist somit eine der aspektuell-lexikalischen Unterscheidung sowie die Markierung der Verbklammer in Vletzt, die Funktion des Verbpräfixes dagegen die der Aspekt- und damit der Diathesenmarkierung.

\subsubsection{Desuffigierung und Deflexion}

Der Schluss im vorigen Abschnitt, dass Derivation (Wortableitung) geringerer Erosion als Flexion ausgesetzt ist, bestätigt sich bei postponierten Morphemen beider Markierungsklassen, d.h. bei Derivation anders als bei Flexion. Man beachte etwa, dass das Nominalabstraktsuffix -the erhalten blieb wie in foul-filthe ,filth-Schmutz", young - you(ng)the ,youth-Jugend", andere freilich darwinschem Wettbewerb erlagen und Alternativen weichen mussten wie etwa -reden und -lac, deren Funktion -ness übernahm. Dagegen ist das Infinitivsuffix geschwunden, allerdings bezeichnenderweise unterschiedlich im funktionalen Wechsel mit der Nominalpräposition to. $-(e) n$ schwand im Infinitiv zum Ende des 14. Jhs. (Lass 1992, S. 98). Muss man 
hier davon sprechen, dass das Englische lernte zu unterspezifizieren oder dass es eine Überspezifikation aufgab? Schon ae. lag funktionaler Unterschied zwischen dem bloßen Infinitiv und der Nominalform mit to (Gerundium) vor. Der bloße Infinitiv kennzeichnete zusammen mit dem einbettenden Prädikat eher transitive Handlungen als Einzelereignis (Fischer 1997). Als das Infinitivsuffix abgebaut wurde und in Konstruktion mit to trat, verwischte sich der ursprüngliche Unterschied keineswegs: mit -ing markierte Verben übernahmen die stärker transitive Beziehung, während Infinitive diese eher aufgaben. Vgl. (13a $\neq b)$ (Fischer 1997, S. 126)

\section{(13) a I saw him doing it \\ b I wanted to do it}

Das Englische verlor allerdings neben seinem Infinitivsignal andere verbkennzeichnende Suffixe wie ae. -sian, -ettan und -locan, die dazu dienten, aus Nomina und Adjektiven Verben zu machen. Vgl. (14).

(14) ricsian „regieren“ zu „reich“, licettan „vortäuschen“ zu „lice; gleich“, geanlacan „vereinigen, angleichen“ (Mitchell/Robinson 1986, S. 60)

Das entspricht im allgemeinen der Konversionspolitik des Neuenglischen schlechthin, nämlich Wortartkonversionen unmarkiert zu lassen - was ja im Deutschen unmöglich ist. Vgl. (15).

(15) deutsch $\left[_{N}\right.$ Telephon $] \neq\left[_{v}\right.$ telephonieren $]$; englisch $\left[_{N}\right.$ telephone $]=\left[_{v}\right.$ tele fone]

Natürlich förderte der Endsilbenschwund - dies nicht nur am Verbinfinitiv - allgemein das Einsilblercharakteristikum des Englischen, das seinerseits die Nullkonversion von $\mathrm{V}$ zu $\mathrm{N}$ und umgekehrt erleichterte.

\subsubsection{Richtungsadverbien - und Aspekt (kein ge- im Englischen und Skandinavischen, Teilabbau im Ndl.!)}

Das Germanische hat allgemein Adverbien zur Bezeichnung der Bewegung zu einem Sprecher oder einem anderen Orientierungspunkt hinzu oder von ihm weg ausgebildet. Vgl. schwedisch här „hier", hit „hierher, herzu“ sowie härifrån ,von hier" oder ae. her, hider, heonan, vgl. Shakespeare hither - thither. Ne. sind sie völlig aufgegeben. Obwohl eine Begriffs- und Verwendungslücke zu bestehen scheint (vgl. ae. heonan $>$ ne. from here), hat bloß das deutsche die ursprünglichen Pronominaladverbien dafür auch behalten. Wir machen in Hinkunft also einen Unterschied zwischen dt. hin(-)/her(-) und den analytischen Formen there to/from there - die ja, wenn wir das deutsche sprecher-origo-orientierte System als Kriterium nehmen, auch nicht dasselbe bedeuten. Abb. 3 ist in diesem Sinne nicht voll repräsentativ. 


\begin{tabular}{|c|c|c|c|c|c|c|c|c|c|c|c|}
\hline & Dt. & Ndl. & Fr. & Afrik. & Schw. & Isl. & Far. & Jidd. & Obdt. & Ae. & $\mathrm{Ne}$. \\
\hline $\begin{array}{l}\text { zum } \\
\text { Origo } \\
\text { hin }\end{array}$ & $\begin{array}{c}\text { hier- } \\
\text { her, } \\
\text { herauf }\end{array}$ & $\begin{array}{c}\text { hier, } \\
\text { (naar- } \\
\text { toe })\end{array}$ & $\begin{array}{c}\text { hjir, } \\
\text { hjir- } \\
\text { hinne }\end{array}$ & $\begin{array}{c}\text { hier } \\
\text { (heir- } \\
\text { natoe) }\end{array}$ & $\begin{array}{c}\text { här, } \\
\text { hit }\end{array}$ & $\begin{array}{l}\text { hér, } \\
\text { hin- } \\
\text { gath }\end{array}$ & $\begin{array}{c}\text { her } \\
\text { higar }\end{array}$ & $\begin{array}{l}\text { hia- } \\
\text { her }\end{array}$ & $\begin{array}{l}\text { auf- } \\
\text { her, } \\
\text { abher }\end{array}$ & $\begin{array}{c}\text { her, } \\
\text { hider }\end{array}$ & here \\
\hline $\begin{array}{l}\text { weg } \\
\text { vom } \\
\text { Origo }\end{array}$ & $\begin{array}{c}\text { da/dort- } \\
\text { hin, } \\
\text { hinauf }\end{array}$ & $\begin{array}{c}\text { daar } \\
\text { (naar- } \\
\text { toe) }\end{array}$ & $\begin{array}{c}\text { dêr, } \\
\text { dêrhin- } \\
\text { né }\end{array}$ & $\begin{array}{l}\text { daar } \\
\text { (daar- } \\
\text { natoe) }\end{array}$ & $\begin{array}{c}d \ddot{a} r, \\
d i t\end{array}$ & $\begin{array}{l}\text { thar, } \\
\text { than- } \\
\text { gath }\end{array}$ & $\begin{array}{c}\text { har } \\
\text { hagar }\end{array}$ & $\begin{array}{l}\text { dort } \\
\text { ahin }\end{array}$ & $\begin{array}{l}\text { auf- } \\
\text { hin, } \\
\text { abhin }\end{array}$ & $\begin{array}{l}\text { thoer, } \\
\text { thider }\end{array}$ & there \\
\hline $\begin{array}{l}\text { w- } \\
\text { For- } \\
\text { men }\end{array}$ & $\begin{array}{l}\text { woher, } \\
\text { wohin }\end{array}$ & $\begin{array}{l}\text { waar, } \\
\text { waar- } \\
\text { heen, } \\
\text { (waar- } \\
\text { naartoe) }\end{array}$ & $\begin{array}{c}\text { wêr, } \\
\text { wêr- } \\
\text { hinne }\end{array}$ & $\begin{array}{c}\text { waar } \\
\text { (waar- } \\
\text { heen) }\end{array}$ & $\begin{array}{l}\text { var, } \\
\text { vart }\end{array}$ & $\begin{array}{l}\text { hvar } \\
\text { hvert }\end{array}$ & hvar & $\begin{array}{c}\text { wu, } \\
\text { wuhin }\end{array}$ & $\begin{array}{c}\text { wo_- } \\
\text { her } \\
\text { wo_- } \\
\text { hin }\end{array}$ & $\begin{array}{c}\text { hwoer, } \\
\text { hwi- } \\
\text { der }\end{array}$ & where \\
\hline
\end{tabular}

Tabelle 3: Richtungsadverbien im Germanischen (nach McWhorter 2001)

Englisches where to? ist allemal where? gewichen. Aber auch in dem Deutschen so verwandten Sprachen wie dem Ndl. herrscht Verschmelzungstendenz: Waar ga je (heen)? Im DaF-Unterricht muss ja in einer Reihe von Fällen jeweils nach Richtungs- oder Statikverortung zwischen Präpositionsakkusativ und Präpositionsdativ entschieden werden - oft keine Kleinigkeit (Abraham 2001). Auffällig ist aber die Sprecherorigo-Orientierung des Deutschen, die in keiner der anderen germanischen Sprachen (im Ndl. auch nur mehr in unsystematischen Spuren) lückenlos vertreten ist.

\subsection{6 sein beim 2. Partizip}

$\mathrm{Zu}$ den auffälligsten Merkmalen der Funktion des Hilfsverb gehört die systematische Trennung zwischen SEIN/ESSE und HABEN/HABERE, die das Germanische und das Romanische teilen: gegessen haben, aber gekommen sein, dies sogar im sonst so vereinfachenden Afrikaans vertrokken zijn (Ponelis 1993, S. 444) und im Jiddischen geblibn zayn (Lockwood 1995, S. 83). Freilich: ein ae. Beispiel wie das folgende will uns angesichts des Deutschen nicht als Nachweis für dieselbe Auxiliar-Strategie erscheinen (ae. beon bzw. wesan):

(16) hu sio lar Lædenge dhiodes ær dhissum afeallen wæs wie das Lernen Latin-Sprache.GEN vor diesem abgefallen war „Wie das Lateinstudium davor verfallen war" (Mitchell/Robinson 1986, S. 111)

Im Altnordischen ebenso wie im modernen Isländischen kennzeichnet das be/sein-Perfekt - durchaus in Entsprechung zum Althochdeutschen bis zum Neuhochdeutschen und im Altenglischen (Mitchell 1985, S. 302-4) - in der Regel das Resultativ sowie das Passiv (Heusler 1950, S. 136). Seine Verwendung als reines Perfekttempus beschränkte sich auf wenige intransitive Verben wie "gehen“: ek em gengenn ,ich bin/*habe gegangen" (Brenner 1882, 
S. 129). Nach Mitchell/Robinsons Zeugnis war aber der Gebrauch von beon und wesan gegenüber habban bereits ae. weithin verwischt. Die Systematik des heutigen Deutschen, nämlich dass SEIN nur bei perfektiven Verben und im Zustandspassiv Verwendung findet, fehlte bereits in den frühesten Stadien der Aufzeichnungen im Englischen. Spätestens im 15. Jh. war be im periphrastischen Perfekt bei den intransitiven Mutativa auf die Verben come, become, arrive, enter, run und grow zusammengeschrumpft (Traugott 1972, S. 144, Rissanen 1999, S. 213). Man vgl. (17).

(17) And didst thou not, when she was gone downstairs, desire me to be no more so familiarity with such poor people? (Henry IV, II.i.96) (nach Traugott 1972)

Und spätestens im 18. Jh. kam das be-Perfekt nur mehr bei become vor (Strang 1970, S. 100), bis es völlig schwand.

Es lässt sich das Argument führen, dass der Abbau von be als Hilfsverb für die Perfektperiphrastik bei Mutativa mit dem Aspektverfall im Englischen und damit der semantischen Klassenselbständigkeit eben jener Mutativa zusammenhängt. Für Aspektverfall bzw. Aktionsartverfall spricht ja das völlige Verschwinden von ge- als Klassenmerkmal der Mutativa ebenso wie am Partizip des Perfekts. Der Zusammenhang zum Schwund der Vorsilben, die zum Teil transitivierende bzw. perfektivierende Wirkung hatten (vgl. dt. be-, ver-), sowie der Schwund des diathetischen Reflexivs als Detransitivierer führt über etwas längere Wege, ist jedoch gleichwohl vorhanden. Klassenbildung, signalisiert über morphosyntaktische Selektion, erleichtert, so haben wir mehrfach argumentiert, vor allem über die Mittelfeldstrecke bis zum lexikalischen Verb in Satzendstellung die Interpretationsarbeit in der linearen Verarbeitung des Satzes. Zudem spielt eine Rolle, dass das Zustandspassiv vom Ae. bis zum Ne. mit dem kanonischen be als Vertreter fürs Passiv schlechthin, also auch für das Vorgangspassiv eingesetzt wurde (Mustanoja 1960, S. 501). Auch hier bietet sich an, das Englische als Minimalisten bezüglich der Balance zwischen Grammatikintension und Diskursaufgaben zu betrachten, zumal ja aus dem Zustandspassiv (mit SEIN/BE) auf den vorhergehenden Passivvorgang bloß geschlossen werden kann, d.h. dass eine notwendige Implikation für den eigentlichen Passierungsvorgang herhalten muss, während das Deutsche - zusammen mit den meisten anderen germanischen Verwandten (nur das Schwedische nimmt sich aus; Holmes/Hinchliffe 1997, S. 100) - Vorgangspassiv und Zustandspassiv morphologisch getrennt kennzeichnet.

(18) a Schwedisch:

Vi har rest till Spanien för

we have travelled to Spain before

„Wir *haben/sind schon einmal nach Spanien gereist" 
b Dänisch:

Barnet er kommen

Kind-das ist gekommen

c Norwegisch:

Han er reist

er ist abgereist

d Isländisch (Kress 1982, S. 148-9):

ég er kominn ich bin gekommen $=$,Ich bin hier“ ég hef komid ich habe gekommen

Zustandsperfekt bei mutativen/resultativen Intransitiva

Vorgangsperfekt bei (In)Transitiva

Schon das Mittelenglische hatte einen weitaus unspezifizierteren Stand bezüglich solcher Unterscheidungen als das Isländische.

Im Deutschen dagegen ebenso wie im Ndl. und Friesischen erhielt sich SEIN sehr konsequent als Hilfsverb (und damit als semantischer Klassenselektor) bei den intransitiven Mutativa sowie bei Fortbewegungs- und manchen Lageverben - im Norddeutschen und Ndl. noch viel konsequenter auf telisch-terminative Verwendungen gemünzt und dort in Variation mit Imperfektivität im Unterschied zum Oberdeutschen. Vgl. (19)-(21).

(19) a Hij is zijn beurs vergeten/verloren er ist seine Börse vergessen/verloren

„Er hat seine Börse nicht bei sich“ (= Resultat mit Implikation „zuhause liegen gelassen")

b Hij heeft zijn beurs vergeten/verloren er hat seine Börse vergessen/verloren „Es passierte ihm, dass er seine Börse vergaß/verlor" (= Vorgang)

(20) a heeft gestaan - hat gestanden (obd. nur ist gestanden), aber is komen te staan ,hat sich hingestellt"

b heeft de hele middag gezwommen „hat den ganzen Nachmittag geschwommen" - is naar het andere oever gezwommen ,ist ans andere Ufer geschwommen"

(21) hat den ganzen Abend getanzt - ist in den Saal hineingetanzt, hat geschlafen - ist eingeschlafen

Damit erweisen sich das Ndl. und das Deutsche (das Norddeutsche konsequenter als das Süddeutsche) klar perfektivsensibel. Ganz deutlich geht es hier nicht um unnötigen Luxus, sondern um ganz wertvolle psychologische Erleichterungen unter der Perspektive der aktuellen linearen Satzverarbeitung - nur unter dieser Perspektive allerdings sind die Vorteile der systematischen Funktionstrennung von SEIN und HABEN in der Verbperiphrase sichtbar zu machen. So ist mit der Setzung des Auxiliars in V2 die Klasse der an SEIN oder HABEN anschließbaren lexikalischen Verben eindeutig einge- 
schränkt, und es dient somit die schrittweise verlaufende Abarbeitung der weiteren Objekte zur massiven Einschränkung der Zahl von erwartbaren Prädikaten in Vletzt derart, dass die semantische Analyse vor dem Erreichen von Vletzt bereits wertvolle Vorarbeit leisten konnte - im Sinne der Parsingstrategie: dass die Zwischenspeicherung des lexikalischen Materials von V2 an bis zur Paenultima vor Vletzt nicht ohne wertvolle Vorarbeit zur Semantik verstrich. Zudem - und auch das ist wertvolle systematische Vernetzung und damit Behaltenserleichterung - steht SEIN nicht nur bei 2. Partizipien resultativer Intransitiva und telischer Verbphrasen, sondern eben auch bei adjektivischen und nominalen Prädikaten. Damit ist die grammatische Synonymie und Klassensemantik von resultativen 2. Partizipien (mit Zustandslesart, egal ob im Passiv oder im Aktiv), Adjektiven und Nomina erreicht, allemal also im semantischen Typus $\langle e,\langle e, \downarrow\rangle\rangle$. Das sind kompensatorische Erleichterungen, die das Englische mit prinzipiellem SVO erst gar nicht braucht - gewiss. Aber das Deutsche, so können wir sagen, schafft sich damit gegen die unvorteilhaften Parsingvoraussetzungen bei Vletzt die starken Diskursvorteile des Scrambling. Dazu im letzten Kapitel. Im Altnordischen wurde das Passiv im allgemeinen mit vera ,sein“" gebildet (zum Suppletivsystem bin-sein-war/gewesen).

\subsubsection{Vorgangspassiv mit werden}

SEIN/ESSE im Selektionskontext mit dem 2. Partizip steht nicht nur mit HABEN/ HABERE in Konstruktionskonkurrenz, sondern auch mit dem inchoativen WER$D E N$ und zwar bei der Bildung des Passivs. Dabei gibt es formal eine interessante Überschneidung. $S E I N+2$. Part. ist bei transitiven resultativen Verben Zustandspassiv, bei intransitiven resultativen Verben dagegen periphrastisches Perfekt. Vgl. (22) im Gegensatz zu (23).

(22) a Der Zahn ist gezogen

b Der Zug ist angekommen als Folge von (23) a *Der Wagen ist gezogen

b Der Zug ist gefahren als Folge von

Der Zahn ist gezogen worden

Der Zug kam an

keine Folge von Wagen ist gezogen worden

keine Folge von Der Zug fuhr

Genau dieses Viererverhältnis ist innerhalb (und außerhalb) der Germania einmalig für das Deutsche. Darüber hinaus hat das Deutsche als einzige Sprache werden fürs Vorgangspassiv und fürs Futur eingeführt, nach dem Zeugnis des Alt- und Mittelhochdeutschen nicht aus eigener Kraft, sondern (wenn wir der Spekulation von Leiss (1989) folgen) durch grammatische Entlehnung aus dem Slawischen. In der Folge hat sich ein solches Vorgangsauxiliar z. T. auch für das Passiv im Skandinavischen ausgebildet, nicht jedoch fürs Futur. Es ist diese spezifische Neuerung des Deutschen - werden als auxiliare Futur- ebenso wie Vorgangspassivstütze -, die das Deutsche un- 
ter sämtlichen seiner germanischen Verwandten (und erst recht im Vergleich mit der Romania) auszeichnet. Für das Futur haben ja sämtliche anderen germanischen Sprachen eine SOLLEN- bzw. WOLLEN- Entsprechung entwickelt, welche auch alle Vorstufen des Nhd. einschließlich des Gotischen verwendet hatten (vgl. Abraham 1987, 1989, 1992). Für das Vorgangspassiv hat sich in den skandinavischen Sprachen bliva ausgebildet; seine Herkunft ist nicht ganz geklärt; zu den Annahmen gehört, dass es zur Zeit der Hansekontakte aus dem Deutschen gecalquet wurde. Ndl. ebenso wie Afrikaans verwenden WORDEN fürs Vorgangspassiv, allerdings nur im einfachen Präteritum. Im periphrastischen Perfekt wird geworden weggelassen.

(24) a ndl. De man werd door de hond gebeten

b De man is door de hond gebeten (*geworden)

c afr. Die trui word gebêre „der Pullover wurde aufgehoben“

d Die trui is gebêre (*geword) „der Pullover ist aufgehoben worden“

Das Schwedische setzt das $B E$-Verb vara im Zustandspassiv, bli dagegen im Vorgangspassiv mit öfters Perfektivaspekt (Holmes/Hinchliffe 1997, S. 109).
a Himlen är täckt av moln Himmel.der ist bedeckt mit Wolken
b Han blev påkörd av en bil er wurde überfahren von einem Auto

Im Altnordischen wurde das Passiv im allgemeinen mit vera „sein" (zum Suppletivsystem bin-sein-war/gewesen). wertha „werden" wurde infolge seiner starken lexikalischen Bedeutung nur gelegentlich für das vollverbale Inchoativum verwendet.

(26) Thar saker skal fyrst doma, er fyrra sumar die Fälle sollte (man) erst beurteilen, die vorigen Sommer varth eige um dømt wurden(=gerieten) nicht zum Urteil „Man soll erst über Fälle urteilen, zu denen man im letzten Sommer kein Urteil erreicht hat". (Heusler 1950, S. 37)

Ae. weorthan schwand nach dem Altenglischen völlig. Auch das Isländische hat verða „werden“ neben vera ,sein“ fürs Passiv erhalten (Kress 1982, S. 150). Vgl. (18) oben.

Die Tabellen 3 und 4 bieten einen Überblick zum oben Diskutierten. 


\begin{tabular}{|l|l|l|l|l|l|l|l|l|l|l|l|l|}
\hline & Dt & Ndl & Jid & Fri & Sw & No & Dä & Is & Fär & Af & Ae & Ne \\
\hline Inhärentreflexiva & & & & & & & & & & & & \\
\hline externer Possessor & & & & & & & & & & & & \\
\hline grammat. Genus & & & & & & & & & & & & \\
\hline Präfixverlust & & & & & & & & & & & & \\
\hline Richtungsadverb & & & & & & & & & & & & \\
\hline SEIN-Perfekt & & & & & & & & & & & & \\
\hline Passiv-WERDEN & & & & & & & & & & & & \\
\hline V2 & & & & & & & & & & & & \\
\hline Indefinitpron. MAN & & & & & & & & & & & & \\
\hline
\end{tabular}

Tabelle 3: Syntaxabbau im Englischen im Vergleich mit anderen germanischen Sprachen (nach McWhorter 2001)

(Dunkeltönung für Erhalt, weiß für Abbau)

\begin{tabular}{|l|l|l|l|l|l|l|l|l|l|l|l|}
\hline & Ne & Fr & Nl & Af & Dt & Ji & Is & Fä & No & Dä & Sw \\
\hline grammatisches Genus & & & & & & & & & & & \\
\hline Kasusmarkierung & & & & & & & & & & & \\
\hline 3. Person V-Morphem & & & & & & & & & & & \\
\hline Sg./Pl. V-Morphem & & & & & & & & & & & \\
\hline Konjunktiv & & & & & & & & & & & \\
\hline st./sw. Verbklassen & & & & & & & & & & & \\
\hline st./sw. Adjektive & & & & & & & & & & & \\
\hline V2 & & & & & & & & & & & \\
\hline lang/kurz-Vokale & & & & & & & & & & & \\
\hline robuster Umlaut & & & & & & & & & & & \\
\hline
\end{tabular}

Tabelle 4: Formenabbau in der Germania (nach Lass 1987)

(Dunkeltönung für Erhalt, weiß für Abbau)

Das Englische vergleicht man traditionell mit dem Ndl. und dem Friesischen. Tabelle 4 jedoch zeigt, dass man ebenso gut die Gegenrechnung auftun könnte, nämlich dass die nordischen Sprachen Parallelen mit dem Englischen gemein haben

\subsection{Primär-typologische Merkmale des Deutschen}

Im Unterschied zur Referenzwiederaufnahme nach verschiedenen Unterscheidungskriterien kann eine Sprache, die notwendigen satzgrammatischen Bedingungen vorausgesetzt, Textverdichtungen und pragmatische Einbindungen von Information zum Sprecher und zu den anderen Redebeteiligten bzw. zum Ablauf der Rede durch bestimmte Wortstellung und Satzakzent vornehmen. Jede Sprache wird dies tun wollen, sie wird dies jedoch nach Maßgabe ihrer satzgrammatischen Möglichkeiten anders tun. Das Japanische z. B. markiert im Text vorweg genannte Satzteile mit eigenen Diskurssuffixen im Unterschied zu neu eingebrachtem Informationsmaterial; das 
Deutsche weiß Satzakzent - genauer: normalen und kontrastiven Satzakzent - zur Erstellung unterschiedlicher Redevoraussetzungen (,Präsuppositionen') ebenso wie Modalpartikel einzusetzen; letztere Möglichkeiten - Modalpartikel zur Signalisierung von Sprecherannahmen zum jeweiligen Textstand - fehlen in den meisten anderen europäischen Sprachen völlig, und dort, wo das Deutsche Akzent und reine Mittelfeldumstellung vornimmt, greifen die germanischen SVO-Sprachen, vornehmlich das Englische, zu eher stark platzkonsumierenden, umständlichen Satzspaltkonstruktionen. Ich reihe solche diskursordnende und textstatusklassifizierende Mittel zu den primären typologischen Merkmalen. Dazu gehören einige morphologisch-paradigmatische Voraussetzungen, die es zuerst hervorzuheben gilt.

\subsubsection{Rektion und Basiswortstellung}

Direkte Grundlage einiger der vergleichend-typologischen Beobachtungen, die zur Charakteristik der besonderen Diskursprominenz des Deutschen führen, sind Kasusrektion und die lineare Abfolge der Rektionsglieder. Das Deutsche ist in dieser Hinsicht nicht nur in der Germania allein stehend, ungeachtet etwa der Tatsache dass auch das Isländische Genitive, Dative und Akkusative besitzt-über ein reicheres Rektionskasusinventarverfügt das Deutsche ja auch nicht. Es spielt aber die Abfolge der Valenzkasus eine entscheidende Rolle, zudem die Verteilung von grammatischem und kontrastivem Akzent, die dabei mit zu Wort kommen. Dies sind die wichtigsten Rektionstypen in ihren Grundabfolgen (Czepluch 1988, Abraham 1995, S. 617 f., Haider 1997).

$\begin{aligned} \text { (28) a zweiwertig: } & \text { NOM }>\text { AKK BEDAUERN, SCHLAGEN } \\ & \text { AKK }>\text { NOM interessieren, imponieren } \\ & \text { NOM }>\text { DAT helfen, gratulieren } \\ & \text { DAT }>\text { NOM gefallen, fehlen } \\ \text { b dreiwertig: } & \text { NOM }>\text { DAT }>\text { AKK anvertrauen, verbieten } \\ & \text { NOM }>\text { AKK }>\text { DAT aussetzen, unterordnen } \\ & \text { NOM }>\text { AKK }>\text { GEN bezichtigen, anklagen } \\ & \text { NOM }>\text { AKK > AKK lehren }\end{aligned}$

Auf Präpositionsrektion verzichte ich hier - nicht ohne Grund, nämlich um später den eingeschränkten Scramblingbegriff einführen zu können. Das Auffälligste und wahrscheinlich in der Schulgrammatik am stärksten Vernachlässigte ist die Abfolge, die ohne Akzentwechsel nicht geändert werden kann. Eindeutige empirische Probe ist neben der Akzentfeststellung der durch Chomsky eingeführte Präsuppositionstest (Chomsky 1976, Höhle 1982).

In den anderen germanischen Sprachen gibt es mit Ausnahme des Isländischen, eingeschränkt auch Ndl. und Friesischen keine morphologische Unterscheidung zwischen Dativ und Akkusativ. Was im Deutschen eine Dativrealisierung hat, wird in den skandinavischen Sprachen in verschiedenen Präpositionsrektionen ausgedrückt. Präpositionslose Dativentsprechungen 
finden sich zuweilen allerdings im Pronominalparadigma, dann aber meist ohne eigene morphologische Unterscheidung vom Akkusativ. Das Allerwesentlichste jedoch ist die Basisabfolge der Kasusrektionsmuster. Diese teilen weder das Isländische noch die engsten Schwestern des Deutschen, das Ndl. und das Friesische und erst recht nicht das Afrikaans oder das Jiddische. Unter den außergermanischen europäischen Sprachen scheinen nur das Finnische und Ungarische das Deutsche an Kasusinventar zu übertreffen. Unter der Hauptperspektive, dass aus diesen Rektionsgrundabfolgen wesentliche Scramblingeigenschaften abzuleiten sind, werden wir jedoch auch diese beiden Sprachen vom Deutschen absondern. Das Deutsche bleibt, was die spezielle Kriteriendurchmischung von Kasusrektion und Abfolge der Valenzglieder betrifft, in einer absoluten Sonderstellung - einer, der überflüssiger Luxus eignet?

\subsubsection{Freie Mittelfeldversetzung der Rektionsglieder (,Scrambling')}

Freie Mittelfeldversetzung (FMV; Scrambling) ist die Versetzung der in $(28 \mathrm{a}, \mathrm{b})$ genannten Rektionspläne innerhalb des Mittelfelds ausschließlich zu Zwecken der Textintegration über den Einzelsatz hinaus. Interessant ist dieser Versetzungsbegriff nur dann, wenn er entscheidend eingeschränkt verwendet wird. Dies sind die 3 Hauptvoraussetzungen des hier verwendeten Scramblingbegriffs (Haider/Rosengren 1998, S. 5; 28):

(29) a morphologisch unterschiedene Valenzrektion;

b eindeutig verschiedene Abfolgebeziehungen im Sinne von (1);

c Mittelfeldausbildung (,MF') zwischen_V[ ${ }_{\mathrm{MF}}$ _]V

Es geht also um den folgenden strukturellen Prozess:

(30) $\mathrm{C}^{0}\left[\mathrm{XP}_{\mathrm{i}} \mathrm{YP}_{\mathrm{j}} \mathrm{V}^{0}\right] \rightarrow \mathrm{C}^{0}\left[\mathrm{YP}_{\mathrm{j}}\left[\mathrm{XP}_{\mathrm{i}} \mathrm{e}_{\mathrm{j}} \mathrm{V}^{0}\right]\right]$

wobei $X P$ und $Y P$ im Valenzmuster von $V^{0}$ und wo $\left[X_{i} Y P_{j} V^{0}\right]$ die Valenzbasisabfolge im Sinne von (29a) ist.

Damit sind folgende Versetzungen ausgeschlossen (z. T. Haider/Rosengren 1998, S. 2-4):

(31) a Argument-Adjunkt-Abfolge wie im Ndl.:

dat Sofie $<$ vandaag $><<$ dat artikel $>><$ vandaag $><<e>>$ moet lezen

b Objektversetzung (-,shift') wie im Isländischen:

Í ger las Jón $<<$ boekurnar $>><e k k i><<e>>$

gestern las J. die Bücher nicht

c T-scrambling ( $=$ Vorversetzung in eine $\mathrm{C}$-adjazente funktionale Spez(Tempus)-Position):

dass $<$ verlieren $>$ Boris nicht $\langle e>$ konnte

*dass Boris $\langle$ verlieren $>$ nicht $\langle e\rangle$ konnte

d Langabstandscrambling wie im Japanischen

e Rechtsherausstellung wie im Hindi oder Türkischen 
f Pronomenversetzung in den Wackernagelbereich (,WP'): dass $I_{\text {WP }}<e s$ ihm $>$ ] Peter $<e>$ doch nicht gezeigt hat

Es gehören nach (29a-c) die folgenden Sprachen aus den folgenden Gründen nicht zu Scramblingsprachen:

(32) a alle festlandskandinavischen Sprachen - Dänisch, Norwegisch, Schwedisch -, die weder das Kriterium von OV (damit kein MF für die nötige Aktantenbeweglichkeit besitzen) noch das der prinzipiell valenzbedingten Basisabfolge erfüllen.

$\mathrm{b}$ das Isländische und das Färöische, die zwar Dativobjektmarkierung aufweisen, aber vom VO-Typus sind und das Kriterium der valenzbedingten Basisabfolge nicht erfüllen - d.h. weniger lexikalische Syntaxvoraussetzungen aufweisen.

c das Niederländische, Friesische sowie das Afrikaans: diese lassen zwar Topikalisierung zu (was mit Scrambling im engeren Sinne nichts zu tun hat, vor allem deshalb, weil alle VO-Sprachen Topikalisierung ebenfalls zulassen und weil dies nicht an die Ausbildung eines Mittelfelds gebunden ist), sie lassen weiter ,ndl. Scrambling“, also ADVDO, DO-OBJ- sowie DO-PP, PP-DO-Abfolgen zu. Aber es zeigen sich keine Beschränkungen hinsichtlich der lexikalisch bedingten Valenzbasisabfolge (schon deshalb, weil ja in keinem Nominal bzw. Pronominalparadigma kasusmorphologische Unterschiede angezeigt sind), und die zwar OV-Charakter (linksläufige Rektion) und auf Pronomina beschränkte Dativpositionen (ohne morphologische Markierungen!) zeigen, aber denen die Kennzeichnung der valenzbedingten Basisabfolge fehlt.

$\mathrm{d}$ das Jiddische: hauptsächlich deswegen weil es keine reine OV-Sprache ist, d.h. weil es IO in linksläufiger, DO jedoch in oberflächlich rechtsläufiger Rektionsstellung (nach Diesing 1997, S. 390) definite NPs in LR, indefinite in RR) hat. Vgl. (33) (im Anschluss an Haider/ Rosengren 1999, S. 78 f., dort Beispiele (107)-(108)) sowie (11a) weiter unten.

e das Englische wegen Nichterfüllung aller dreier Kriterien: VO (damit kein Mittelfeld), kein Dativ, damit auch keine irgendwie interessante Erfüllung des Kriteriums der valenzbedingten Basisabfolge.

Dem Jiddischen kommt wegen seiner Herkunft aus dem Oberdeutschen unser besonderes Augenmerk $\mathrm{za}$ - das Oberdeutsche ist ja eine ausgesprochene Scramblingsprache - und damit eine eminent diskursprominente Sprache. Man beachte, dass sich im Jiddischen kein Mittelfeld zur Beortung freier Mittelfeldversetzung ausbildet. Vgl. (33).

(33) a Max hot nit gegebn Rifken dos buch

b Max hot $<$ Rifken $>$ nit gegebn $<e>$ dos buch

c Max hot $<$ Rifken $>$ nit gegebn $\langle e>$ dos buch 
d Max hot $<<$ dos buch $>><$ Rifken $>$ nit gegebn $<e><<e>>$

e Max hot [<Rifken $>$ nit $[<<$ gegebn $>>[<$ e $>$ ken buch $<<$ e $>>]]]$

f Max hot $[<$ dos buch $>$ nit $[<<$ gegebn $>>$ [ken jingl $<\mathrm{e}><<\mathrm{e}>>]]]$

\subsubsection{Verbklammer (SVOV), strenge Verbzweitstellung und Parsing}

Alle germanischen Sprachen außer dem Englischen - auch das sonst so oft abweichende Afrikaans-zeigen V2-Wortstellung in dem Sinne, dass auch Nichtsubjekte vor dem Verb stehen können. Dazu stellen sich asymmetrische Verbpositionen je nachdem, ob der Satz abhängig oder eingebettet ist: die skandinavischen Sprachen zeigen dabei leichte Wortstellungsveränderungen zwischen Verb und Satzadverb, obwohl sie das Verb - im Unterschied zum Altnordischen, dem Altdänischen und Altnordwestnorwegischen (Propst 2002) im abhängigen Satz nie in die letzte Position rücken. Dies tun nur die drei festlandwestgermanischen Sprachen: das Deutsche, das Niederländische und das Westfriesische.Ae. hatte wie das moderne Deutsch, wenn auch nicht so streng, SVOV, genauer_V_V. Dieses_V_V beginnt seinen Abgesang im Englischen im 15. Jh. und ist im 17. Jh. völlig verschwunden. Die Frage, warum diese Struktureigenart eintreten musste, wird auch Licht auf die Frage werfen, warum sie im Deutschen nicht nur erhalten blieb, sondern sich im Gegenteil immer strenger ausbildete (z. B. verschwand älteres V3).

Eine der Annahmen ist, dass das flektierte Verb zu C(omp) in komplementärer Verteilung steht und dass diese Komplementarität dann verloren gehen muss, wenn die Kasusmorphologie erodiert und Kasus durch lineare Stellung und durch Präposition identifizierbar werden muss. Ein gelernter Germanist braucht sich zur Widerlegung dieser These nicht erst durch die lange und komplizierte Literatur von Platzack/Holmberg (1989) über Vikner (1995) bis Kroch/Taylor (1997), Bobalijk/Thráinsson (1997) und Rohrbacher (1999) - vor allem die frustrierenden Parameter starker und schwacher Flexion - hindurchzuarbeiten. Ihm genügt das Zeugnis der deutschen Dialekte, in denen Kasusunterschiede weithin geschwunden sind, V2 und Vletzt jedoch ungebrochen erhalten geblieben sind. Man beachte jedoch, dass auch die Annahme, dass nach dem Zeugnis von Pidgin- und Kreolsprachen (Heine 1975, Kouwenberg 1994, Baptista 2000) sowie der Interimsprache beim Erstspracherwerb reiner SOV-Sprachen (Clahsen/Muysken 1986) SVO die universal unmarkierte Satzgliedfolge ist, weder die Frage beantwortet ist, warum das Englische [_V_] aufgegeben hat, die anderen germanischen Sprachen es unter Einschränkungen behalten haben und nur die Festlandwestgermania es durch [_V_V] ersetzt hat, wobei das Deutsche am konsequentesten war. Dies freilich fordert wiederum eine Antwort auf die Frage, warum SVO unter den Weltsprachen so weit und offenbar präferiert verbreitet ist.

Sowohl die bevorzugte Ausbildung in Pidgins und Kreolsprachen-übrigens unabhängig vom typologischen Muster der Substratsprache (das ndl. Berbicekreol, das sich zwischen Sprechern des Ndl. und der SOV Niger-Congo-Sprache Ijo ausbildete) - als auch das Zweitspracherwerbsargument von Clahsen/ 
Muysken (1986): SVO als Zwischenkompetenz bei Deutsch-Türkisch-Lernern) weisen stark auf Druck unter Parsingbedingungen hin. Ich werde diese These in Kapitel 5 unten zur Diskursprominenz des Deutschen entwickeln.

Hier sei nur in Kürze auf Erscheinungen in den gesprochenen Varianten des Deutschen verwiesen, die auf Parsingstrategien zurückgehen: Konjunktionsflexion in den bairisch-österreichischen Mundarten; Abfolge der Verben im V-Komplex (unter den verbleibenden OV-Sprachen zeigt nur das Zeitungsfriesische strenges, von der Grammatiklogik diktiertes Vletzt - siehe Abraham 1995); Perfektperiphrase für die einzige Präteritumsfunktion; Flexionsunterscheidung von Definitheit und Indefinitheit im adjektivischen Attributkomplex - und letztlich auch V2 (als Erklärung für das große V2-Überwiegen in den Sprachen der Welt ${ }^{7}$ ).

Wie sehr andererseits Rektionslinksläufigkeit im Deutschen verankert ist, bestätigt eindrucksvoll die linksläufige Rektionseinbettung im Attributkomplex sowohl bei Adjektiven als auch bei Verbpartizipien. An solchen Komplexen scheitert bereits das dem Deutschen so eng verwandte und sonst so gleichlaufende Niederländische.

Tabelle 5 vermittelt wieder einen Überblick.

\begin{tabular}{|l|l|l|l|l|l|}
\hline & Ne. & Afrik. & Jidd. & Dt. & Nld. \\
\hline einfaches Präteritum & & & & & \\
\hline eigenes Infinitivsuffix & & & & & \\
\hline Verbklammer[[FR 8]] & & & & & \\
\hline OV zugunsten von VO & & & & & \\
\hline Genusklassifikation & & & & & \\
\hline Finitflexion bei V & & & & & \\
\hline attributive Adjektivflexion & & & & & \\
\hline
\end{tabular}

Tabelle 5: Flexionsabbau in den „kontaktschweren“ germanischen Sprachen (nach Lass 1987)

(Dunkeltönung für Abbau, weiß für Erhalt)

${ }^{7}$ Im Anschluss an die Beobachtung, dass sich V2 am längsten in Dokumenten des Kentischen erhält, während es im übrigen Englischen abgebaut wird, gehen Kroch/Taylor (1997, S. 312) davon aus, dass der Abbau von SVOV durch das Skandinavische ausgelöst wurde, dass somit der Übergang vom ,symmetrischen “ (Verbversetzung sowohl in unabhängigen wie in eingebetteten, abhängigen Sätzen) zum asymmetrischen Versetzungstypus (Verbversetzung nur im unabhängigen Satz) zu V2 als Folge des Flexionsverlustes eintrat, dem die frühen skandinavischen Einwanderer in ihrem unvollkommenen Erwerb des Englischen unterlagen (Kroch/Taylor 1997, S. 318-20). Unter der Annahme, dass vollständiger Abbau von V2 nur mit massivem Flexionsverlust einhergeht, impliziert diese Annahme von Kroch/Taylor, dass der Übergang zum asymmetrischen V2 ein Übergangsstadium zwischen der ursprünglichen Konfiguration und der des Neuenglischen war. Ich führe dies hier an, um zu zeigen, welch komplexe, im übrigen völlig undokumentierte Vorannahmen zu solchen Erklärgängen - Kaynes spekulative, empirisch nicht nachvollziehbare Asymmetriehypothese ist ja auch noch vorausgesetzt - nötig sind.

${ }^{8}$ Dies ist auf die Mittelfeldfüllung zwischen den beiden Verbklammern durch Objekte und Adverbien gemünzt: vgl. Ich habe den Brief gestern geschrieben. 
Englisch und Afrikaans sind unter allen einander am ähnlichsten, wiewohl eigentlich - was hier nicht in den Blick gerät - das Afrikaans inbezug auf Morphologie weniger abgebaut hat als das Englische.

\section{Folgerungen}

\subsection{Beschreibung und Erklärung - Englisch $\neq$ Deutsch: Schlüsse fürs Deutsche?}

Die vor allem in der Literatur zum Englischen oft getroffene Erwägung, das Englische habe in seiner Geschichte die Präfixmorpheme deshalb abgelegt, weil sich die Akzentverhältnisse so drastisch änderten (Visser 1963, S. 127), ist nicht haltbar. Zum einen weist das Deutsche denselben Primärakzent auf wie das Englische - jene romanischen lexikalischen Übernahmen, die Endbetonung aufweisen, können ja den Präfixabfall nicht allgemein begründen, da sich die Verben germanischen und romanischen Ursprungs die Waage halten und da ja auch romanische Verben Ableitungsmorpheme in präfigierter Form zeigen. Zudem hatten sich Reflexe des lexikalisch-perfektivierenden und perfektpartizipialen ge- lange in südwestliche Dialektregionen der britischen Insel als $y$ - and $a$ - erhalten (vgl. Barnes 1886, S. 27-8 zum Dorset), bis sie dem Druck der Hochsprache zum Opfer fielen.

\subsection{Was ist nun konkret überspezifiziert?}

Es ist kein Streitpunkt, dass manche Sprachen spezifischer sind als andere. Wohl herrscht auch die Ansicht, dass die Komplexitäten der verschiedenen Sprachen einander über alle Kategorien und Paradigmen hinweg ungefähr die Waage halten (so etwa Edwards 1994, S. 90; Bickerton 1995, S. 67; O'Grady et al. 1997, S. 6; Crystal 1987, S. 6-7). Freilich ist das nirgends erschöpfend untersucht worden. Zuweilen findet sich die Position, dass alle Sprachen komplex sind, freilich manche mehr als andere (Crowley 2000, Gil 2001). Zu den am wenigsten spezifischen Sprachen der Germania gehört das Englische nach dem Vergleich der sekundären und primären Vergleichskriterien.

Natürlich hat das Englische auch komplexe Eigenschaften entwickelt, mit denen es in der Germania sogar alleine steht: etwa die do-Stütze, die Kennzeichnung des unmittelbaren Futurs und präsentischer Imperfektivität mittels der ing-Form - was ja für das einfache Präsens den Weg für Habitualität freimacht.

\section{Was bezweckt die, formal redundante ${ }^{\natural}$ Eigenständigkeit des Deutschen? \\ Exaptation ursprünglicher Gartenpfaderscheinungen oder teleologische Adaptation im Sinne von Parsing- und Diskurserleichterung?}

Man braucht sich der Alternative in dem Kapiteltitel gar nicht zu stellen sie wird gar nicht entscheidbar sein. Aber sie bietet sich nach der hier 
eingeschlagenen Argumentationsrichtung, leichte' oder ,schwere' Sprache einfach an. Ich werde im Folgenden zwei thematische Stoßrichtungen verfolgen: einmal soll gezeigt werden, was die formale Redundanz an strukturellen Möglichkeiten eröffnet, was in anderen germanischen (und außergermanischen) Sprachen nicht gelingt; und zweitens soll begründet werden, inwiefern das Deutsche mit seinem evolutionären Kurs der formalen Redundanz, den keine andere Sprache teilt, eine ,leichte' Sprache ist - allerdings eben nicht für denjenigen, der das Deutsche erst zu lernen hat, also vor allem für solche Lerner, die von SVO und geringerer Sensibilität für morphosyntaktische Desambiguierungen ausgehen. Aus dieser Begründung leitet sich nahtlos ein eindeutiges Plädoyer für das Studium des Lateinischen und Altgriechischen $a b$ - in dem Sinne nämlich, dass keine andere Sprache so sehr auf morphosyntaktische Sensibilisierung und semantische Desambiguierungen sowie Desambiguierungen von Textreferenz vorbereiten wie gerade diese beiden Sprachen.

Bei der Diskussion des massiven Formenabbaus und der darauf folgenden funktionalen Verluste in der Geschichte des Englischen folgert McWhorter (2001), dass die meisten Veränderungen bereits vorlagen, als sich die skandinavischen Stämme in Nordengland und Schotland ansiedelten - dass sie sich somit nicht erst als Folge von Sprachmischung unter Kreolisierung einstellten, dass also im Englischen bereits früh und autonom ,Entspezifizierung' bzw. ,Redundanzabbau' vorlagen. Aber er verbindet damit die Frage, warum nicht ähnliche Abbauprozesse unter dem Druck von funktionaler Entspezifizierung und Redundanzabbau nicht auch in den anderen germanischen Sprachen eintraten. Sollte es so etwas wie „offene Grammatikräume“ in der Syntax ebenso wie in den phonetischen Inventaren geben, wie Visser vermutet (Visser 1963, S. 135), dann wäre nicht gut erklärlich, wieso sich die Pronomina des Englischen nicht Exaptationsprozessen beugten - nach der der evolutionären Biologie entlehnten Idee bei Lass (1990) -, wonach Reflexivpronomina als morphologische Valenz- und Modussignale wie in Dutzenden anderer europäischer Sprachen und besonders im Deutschen und Skandinavischen (nicht jedoch im Ndl. und Friesischen) ausgebildet wurden (McWhorter 2001).

Ich schlage vor, derartige historisch als Exaptationen erklärbare Prozesse daraufhin zu prüfen, ob zwingende innergrammatische (, universalgrammatische') Zusammenhänge vorliegen, die zufällige von systematischen UG-logischen Exaptationen unterscheiden. Als Kandidaten für Syntaxklasseme im zweiten, systematischen Sinne sehe ich

(i) die Reflexivverwendung zur Diathesenanzeige (Mittel- und Passivkonstruktion) sofern man zwischen lexikalischer und syntaktischer Reflexivität trennt bzw. sofern man ins Lexikon ebenso viel Syntaktisches hineinsteckt wie in die wirkliche Syntax. Man denke an das s-Passiv im Skandinavischen und in den slawischen Sprachen. 
(ii) die dreifache Genusklassifikation und -anzeige am Artikelwort und attributiven Adjektiv im Deutschen - natürlich im besonderen nur dann, wenn Diskursstrukturierung und Referenzwiederaufnahme als authentische Grammatikkomponenten gesehen werden und nicht als Erscheinungen einer unsystematischen Pragmatik.

Es ist zum Abschluss dieses ersten Kapitels nochmals zu beleuchten, worauf es bisher ankam. Es galt nicht nur zu zeigen, dass das Deutsche bei jedem der vorgelegten morphologischen Auszeichnungen eine reichere Auswahl trifft als seine germanischen Verwandten, sondern vor allem dass das Neuenglische mit weit sparsameren formalen Mitteln auskommt. Wir leiten daraus das Charakteristikum ab, das Deutsche habe seine Überspezifikation behalten - zu welchem Zweck, das soll jetzt beleuchtet werden.

\subsection{Freie Mittelfeldversetzung und die Thema-Rhema-Struktur}

Im Folgenden sollen die diskursvernetzenden Strategien des Deutschen illustriert werden, die sich aus der Weitung des Mittelfelds - der strukturellen Periode zwischen den beiden Verbklammern, $\mathrm{C}^{\circ}$ (V2) und $\mathrm{V}^{\circ}$ (Vletzt) - anbieten. Man vgl. zuerst die Satzfeldillustrationen in Tab. 6.

\begin{tabular}{|c|c|c|c|c|c|c|c|c|}
\hline & & $\begin{array}{l}\text { THEMA/ } \\
\text { RHEMA }\end{array}$ & - & THEMA & $\leftarrow$ RHEMA & RHEMA & RHEMA $\rightarrow$ & THEMA \\
\hline & KOORD & $\begin{array}{l}\mathrm{L}_{\mathrm{cp} / \mathrm{IP}} \\
\mathrm{SpezCp} / \mathrm{IP}\end{array}$ & Comp/Infl & AgrOP & $L_{v p}$ & $L_{v p}$ & $\left.\left.\left.\mathrm{~V}^{\circ}\right]\right]\right]$ & $\begin{array}{l}\text { Extra- } \\
\text { position }\end{array}$ \\
\hline $\mathrm{i}$ & - & $D u$ & schlägst & den Teig & aber & HART & & heute! \\
\hline ii & Aber & - & - & den Teig & auch & HART & schlagen! & - \\
\hline iii & - & Wieso & - & den Teig & denn & HART & schlagen? & - \\
\hline iv & Aber & wieso & - & den Teig & denn & HART & schlagen & heute? \\
\hline
\end{tabular}

Tabelle 6: Satzfeld: UG-Satzstruktur und die Verteilung von Diskurskategorien [,THEMA/RHEMA " in der 3 . Spalte bedeutet, dass topikalisierte Satzglieder sowohl Thema- wie auch Rhemafunktion gleichzeitig erfüllen können.]

Die folgenden Verteilungsbeziehungen gelten zwischen den Feldpositionen und Akzentunterscheidungen (Cinque 1993):

(34) a Wir haben sie gestern GESEHEN

... GA

b *Wir haben gestern sie gesehen

$\ldots-$

c Wir haben gestern SIE gesehen

... KA

d SIE haben wir gestern gesehen

... KA

e *Sie haben wir gestern gesehen 
Die ungrammatischen Versionen - d.h. solche ohne GA/KA-Zuweisung lassen sich direkt aus Cinques GA-Linking-Regel ableiten (Cinque 1993).

\begin{tabular}{|c|c|c|c|c|c|c|c|c|c|c|}
\hline & & $\begin{array}{l}\text { THEMA } \\
\text { RHEMA }\end{array}$ & - & $\begin{array}{l}\leftarrow \\
\text { THEMA }\end{array}$ & $\begin{array}{l}\text { THEMA } \\
\rightarrow\end{array}$ & $\begin{array}{l}\leftarrow \\
\text { RHEMA }\end{array}$ & RHEMA & $\begin{array}{l}\text { RHEMA } \\
\rightarrow\end{array}$ & THEMA & $\begin{array}{l}G A \\
\neq\end{array}$ \\
\hline & Koord & $\begin{array}{l}\text { [ }_{\text {c/P/P }} \text { Spez } \\
\text { CP/IP }\end{array}$ & $\mathrm{C}^{\circ} / \mathrm{I}^{\circ}$ & WP & AgrOP & $q_{v p}$ & $\int_{\mathrm{vp}}$ & $\left.\left.\mathrm{V}^{0}\right] \mathrm{l}\right]$ & $\begin{array}{l}\text { Extra- } \\
\text { position }\end{array}$ & \\
\hline i & - & $D u$ & wirst & $i h n / ' n$ & - & heute & pünktlich & SEHEN & heute! & GA \\
\hline ii & Aber & - & - & 's 'm & - & auch & pünktlich & ABGEBEN! & & GA \\
\hline$i i i$ & - & Wieso & - & es $i h m$ & - & denn & pünktlich & ABGEBEN? & & GA \\
\hline iv & Aber & wieso & & 'm 's & - & denn & sofort & ZEIGEN & heute? & GA \\
\hline
\end{tabular}

Tabelle 7: Satzfeld: Verbklammer, Diskurskategorien und die Verteilung von grammatischem Normalakzent (GA)

[WP = Wackernagelposition für schwache Pronomina und deren klitische Reduktionsformen; GA = grammatischer Normalakzent, KA = Kontrastakzent]

Die Illustrationen in (35) führen intransitive und transitive Präsentationssätze ein.

(35) a Es übertölpelte ${ }_{{ }_{A D V} / \mathrm{vP}}$ prinzipiell $\left[_{\mathrm{VP}}\right.$ der IGEL den HASEN $\left.\mathrm{t}_{\mathrm{i}}\right]$

b Aber es übertölpeln ${ }_{\mathrm{i}}\left[_{\mathrm{ADV} / \mathrm{vp}}\right.$ jedesmal $\left[_{\mathrm{vp}}\right.$ IGEL HASEN $\left.\mathrm{t}_{\mathrm{i}}\right]$

\begin{tabular}{|c|c|c|c|c|c|c|c|c|}
\hline & - & - & - & - & $\begin{array}{l}\leftarrow \\
\text { Rhema }\end{array}$ & Rhema & Rhema & $\underset{\rightarrow}{\text { Rhema }}$ \\
\hline & Koord & $\begin{array}{l}{\left[\mathrm{l}_{\mathrm{CP}}\right.} \\
\text { SpezCP }\end{array}$ & $\mathrm{C}^{\circ}$ & $\begin{array}{l}{\left[_{1 P}\right.} \\
\text { SpezIP }\end{array}$ & Infl & $I_{v p}$ & {$\left[_{\mathrm{vp}}\right.$} & $\left.\left.\mathrm{V}^{\circ}\right]\right]$ \\
\hline (26a) & - & - & - & Es & übertölpelte $_{i}$ & prinzipiell & der IGEL den HASEN & $t_{i}$ \\
\hline (26b) & Aber & - & - & es & übertölpeln & jedesmal & IGEL HASEN & $t_{i}$ \\
\hline
\end{tabular}

Tabelle 8: thetische (= allrhematische) Satzmuster im Feldverteilungsmuster

Beispiele mit Minimalfokus lassen sich durch die Fragen (36)-(39) elizitieren; die Versetzungen beziehen sich jeweils auf die Basisabfolgen, die durch die Minimalvalenzmuster (Infinitivprädikat mit seinen Objekten und eventuell Lokaladverbien; vgl. *(Mutter den Kuchen) überreichen; *(in der Stadt) wohnen; * (in den Graben) springen) vorgegeben sind.

In den folgenden Mustern zur Verteilung und Beziehung zwischen Fokus (Rhema) und Themagliedern (,Backgrounding') verwende ich die folgenden 
Abkürzungen: $1=$ Nominativ, $3=$ Dativ, $4=$ Akkusativ, $B=$ =Backgrounddiskursfunktion (=Thema), $\mathrm{F}=$ Fokusdiskursfunktion (=Rhema); KAPITÄLCHEN bezeichnen Satzkernakzent; Fokus-F in der Basisposition des minimalen Thetamusters bleiben ohne Fetthervorhebung, Scrambling der Fokusposition wird dagegen durch Fettsatz hervorgehoben; Kursive in der Rechtsrandspalte signalisieren Basisabfolge der Verbargumente.

Textverankerungen der verschiedenen Stellungs- und Akzentverteilungsmuster - vgl. (36)-(39):

(36) WAS-F4 hat der Sohn-B1 Mutter-B3 gestern überreicht?

a Gestern hat der Sohn Mutter den Kuchen überreicht.

$\mathrm{b}{ }^{*}$ Gestern hat der Sohn den Kuchen Mutter überreicht.

(37) WEM hat der Sohn gestern den Kuchen überreicht?

$$
\begin{aligned}
& F 4>B 1>B 3 \text { ? } \\
& \mathrm{B} 1>\mathrm{B} 3>\mathrm{F} 4 \\
& { }^{*} \mathrm{~B} 1>\mathrm{F} 4>\mathrm{B} 3 \quad=\text { Ne.! } \\
& F 3>B I>B 4 \text { ? } \\
& \mathrm{Bl}>\mathrm{F} 3>\mathrm{B} 4 \quad \neq^{*} \text { Ne. }
\end{aligned}
$$

a Gestern hat der Sohn MutTER den Kuchen überreicht

$\mathrm{b}$ Gestern hat der Sohn den Kuchen MUTTER überreicht

c *Gestern hat den Kuchen der Sohn Mutter überreicht

d *Gestern hat den Kuchen MUTTer der Sohn überreicht? ${ }^{*}$ B4> F3>B1 $\neq^{*}$ Ne.

(38) WER hat gestern Mutter den Kuchen überreicht?

a Gestern hat der SoHN Mutter den Kuchen überreicht

b 'Gestern hat den Kuchen der SoHN Mutter überreicht

c 'Gestern hat Mutter der SoHN den Kuchen überreicht

d 'Gestern hat den Kuchen der SoHN Mutter überreicht

e *Gestern hat der SoHN den Kuchen Mutter überreicht

(39) WAS hat sich gestern mit dem Sohn, Mutter und dem Sohn ereignet? V im Fokus
a Gestern hat der Sohn Muttern den Kuchen überreicht
$B I>B 3>B 4>$ F-V?
b *Gestern hat den Kuchen der Sohn Muttern überREICHT
$\mathrm{B} 4>\mathrm{B} 1>\mathrm{B} 3$
c 'Gestern hat Muttern der Sohn den Kuchen überReICHT
B $4>\mathrm{B} 1>\mathrm{B} 3 \quad \neq^{*}$ Ne.
d "Gestern hat Muttern den Kuchen der Sohn überREICHT
B $3>\mathrm{B} 4>\mathrm{Bl} \quad \neq^{*} \mathrm{Ne}$.
e 'Gestern hat der Sohn den Kuchen Muttern überREICHT
'B1 $>$ B4 $>$ B3 $\neq *$ Ne.

$\mathrm{Zu}$ beachten bei der folgenden Generalisierung der Distributionen in (36)(39) ist der Umstand, dass wir nur definite NP-Referenzen eingebracht haben. Bei Indefinit-NPs oder Mischung der Referenz hätten sich andere Ergebnisse eingestellt. Dies sind die Folgerungen - eine entscheidende Bewertungsrolle spielt die Basisabfolge im Valenzmuster (zur Bedeutung dieses Kriteriums gerade für generative Beschreibungen und UG-Ableitungen vgl. Abraham 1997b):

(40) a Wo kein [def]-Unterschied bei den Verbargumenten getroffen wird, da spielt die Basisabfolge des Minimalverbmusters die entscheidende Rolle; man vergleiche (36a)-besser-als-(36b): in (36a) bleibt sowohl die Basisabfolge als auch die grundlegende Abfolge , $\mathrm{B}>\mathrm{F}^{6}$ (Thema-vor-Rhema-Abfolge) erhalten. 
b (36a)-besser-als-(36c), da die lexikalisch vorgegebene Basisabfolge die Generalisierung B $>F$ (,Thema-vor-Rhema') überschreibt.

c Erhalt von B3>B4 ist als Subkonstituente in allen Fällen verständniserleichternd, egal wo B1 steht - es darf eben nur nicht zwischen B3 und $\mathrm{B} 4$ stehen.

(40a) beschreibt auch eine Möglichkeit im Englischen, (40b) dagegen ist im Englischen ausgeschlossen. Im Englischen hat (40c) deshalb keine prominente Rolle zu spielen, weil von vornherein nur SVIODO bzw. SVDOPO erlaubt ist.

Bei Doppelfokusmustern lassen sich identische Generalisierungen treffen.

(41) WER hat denn gestern WAS Muttern überreicht?

a Gestern hat der SoHn Muttern den KuCHEN überreicht F1>B3>F4 $\quad \neq^{*} \mathrm{Ne}$.

b *Gestern hat den Kuchen Muttern der SoHN überreicht ${ }^{*} \mathrm{~F} 4>\mathrm{B} 3>\mathrm{F} 1 \quad \neq^{*} \mathrm{Ne}$.

Das Kriterium der Basisabfolge hat eindeutig Vorrang wie in (41a). Ein weiteres besonderes Ergebnis liefert jedoch der Vergleich der Prädikatsperiphrase in (39a-e) insofern, als die Entsprechungen mit dem simplen, keine sichtbare Verbklammer öffnenden finiten Prädikat. Vgl. (42a-e), wo ausschließlich (a) einigermaßen akzeptabel ist, wenn auch selbst dieses keinesfalls unmarkiert.

(42) WaS ereignete sich gestern mit dem Sohn, Mutter und dem Sohn?

$V$ im Fokus

a 'Gestern überReichte der Sohn Muttern den Kuchen

${ }^{?} \mathrm{~B} 1>\mathrm{B} 3>\mathrm{B} 4$

b *Gestern überreichte den Kuchen der Sohn Muttern

${ }^{*} \mathrm{~B} 4>\mathrm{B} 1>\mathrm{B} 3$

c * Gestern überReichTE Muttern der Sohn den Kuchen

$* \mathrm{~B} 4>\mathrm{B} 1>\mathrm{B} 3$

d *Gestern überreichte Muttern den Kuchen der Sohn

${ }^{*} \mathrm{~B} 3>\mathrm{B} 4>\mathrm{B} 1$

e *Gestern überreichte der Sohn den Kuchen Muttern

$* \mathrm{~B} 1>\mathrm{B} 4>\mathrm{B} 3$

Dies spricht eindeutig dafür, dass die Vletzt-Stellung für das lexikalische Prädikat, damit die Periphrase des Deutschen unter Diskursverteilungskriterien eine hervorragende Rolle spielt. Dies eröffnet für den Präteritumschwund und die das einfache Präteritum ersetzende Periphrastik (in allen Tempora!) eine ganz andere Erklärung als die in der Germanistiktradition kanonisch gehandelte (vgl. Abraham/Conradie 2001).

\section{Genus-, numerus und kasusunabhängige Flexion am attributiven Adjektiv: starke, gemischte und schwache Beugung - reiner Luxus? Ärgerliche Komplizierung? Parsing und die ,unendlichen' Attributketten im Deut- schen.}

Es gibt keine andere Sprache, die wie das Deutsche die Kongruenzmorphologie am attributiven Adjektiv - über Kongruenz nach Numerus, Genus und Kasus - nach referentiellen Kriterien bestimmt, die durch die Klassenzuge- 
hörigkeit der Elemente vor dem Attribut festgelegt werden - was man in der deutschen Grammatikterminologie ,starke‘ und ,schwache' Beugung am attributiven Adjektiv nennt. Nur das Ndl. zeigt noch schwach und unsystematisch Spuren solcher Kongruenz. Ohne auf Einzelheiten einzugehen möchte ich nur die sich ergebenden Folgen hervorheben. Eine Aufgabe (übrigens aus meiner langjährigen DaF-Lehre geholt) möge dies verdeutlichen: es war in (50) die richtige Flexionskette zu erstellen.

(50) a zu eu(r)er all(er) großer/n Schande =

$\mathrm{b}$ tot grote schande van jullie allen

c til stor skam for dykk alle

d ta grutte skande fan jim(me) allegearre ${ }^{10}$

„zur großen Schande von euch allen“

Es ist illustrativ sich den Abarbeitungsprozess von (50a) beim Hörer vor Augen zu führen. Dieser muss in den folgenden Schritten ablaufen:

$1 z u$ fordert den Dativ an Schande - eu(r)er all(er) großer/n bleibt unentschieden, wird zwischengespeichert

2 eu(r)er wird als Possessivpronomen zu Schande identifiziert: damit wird entschieden zu eurer großen Schande

3 aufgrund von Schritt 2 kann aber das nächste Element, all(er), nicht entschieden werden: die Kette all(er) großer/n Schande ist bei jeder morphosyntaktischen Alternative ungrammatisch, da semantisch uninterpretierbar. D.h. Schritt 2 muss zurückgenommen werden. Wir müssen von der Selektion des Quantors all(er) ausgehen und zusätzlich eu(r)er und gro$\beta e r / n$ unentschieden im Zwischenspeicher ablegen.

4 all-kann ausschließlich ein Zählnomen quantifizieren - dabei fällt Schande aus; es bleibt $e u(r) e r$; da das Possessiv kein Zählnomen ist, bleibt die Form des Personalpronomens euer, die als Personalpronomen identifiziert im entsprechenden Paradigma als Genitiv freigegeben wird. Damit ist auch aller in Kongruenz mit euer als Genitiv einzuordnen.

Erst jetzt kann also die Abarbeitung von links nach rechts - hinter der Präposition $z \boldsymbol{u}$ - wieder neu aufgenommen werden.

5 Erst jetzt kann das attributive Adjektiv großer/n vom Zwischenspeicher zur weiteren Parsingoperation freigegeben werden. Es wird im allgemeinen jedoch nicht entschieden werden können, in welcher Artikelkategorie euer aller steht, d.h. ob starke oder schwache Beugung eingesetzt werden muss: zu euer aller großer oder großen Schande. Offenbar ist euer aller zu komplex für eine solche Entscheidung.

9 Jan Terje Faarlund, Oslo, sieht auch die Möglichkeit til dykkar/dere(s) alles store skam, die aber hoch stilisiert sei.

${ }^{10}$ Dank an Tette Hofstra, Groningen. 
Die verzögerte Abarbeitungskette (= Verständnisprozess) zeigt uns zweierlei: einmal dass reiche morphologische Unterscheidungen vorliegen müssen, um überhaupt zu Entscheidungen und damit semantischen Interpretationen vordringen zu können; und zum anderen dass erst damit die Linksläufigkeit - und aufgrund solcher morphologiereicher Unterscheidungen strenge Rechtsköpfigkeit - des Deutschen möglich wird. Und das ist der entscheidende Vorteil des Deutschen: Komplemente wie Modifikatoren können im Sinne des Skopusaufbaus von links-kopfdistal fortschreitend nach rechtskopfproximal ohne Strategiewechsel gesetzt und abgearbeitet werden. Dieselbe strenge Rechtsköpfigkeit herrschte ja auch in der Satzstruktur. Da zudem ein wesentlicher Diskursfunktionsunterschied zwischen Attributen und Prädikaten besteht - Attribute sind unhervorgehoben thematisch/background, Prädikate dagegen rhematisch/fokussiert - erwächst mit den ,unendlichen ${ }^{6}$ Attributketten ein bedeutsamer diskursfunktionaler Vorteil, den keine andere germanische Sprache - und erst recht nicht die reinen SVO-Sprachen der Romania - aufwiesen (ähnlich Fabricius-Hansen 2002).

Auf eine weitere Folge der bei SVOV-Sprachen erforderlichen Parsingstrategien sei kurz hingewiesen. Sowohl Vletzt im finiten abhängigen Satz wie auch die absolute Rechtsköpfigkeit im Nominalattribut wie in (51) erfordern massive Zwischenspeicherleistungen beim Deutschsprecher.

(51) der heute morgen in der Vollversammlung gegenüber den internationalen Abgeordneten durch Kofi Anan auf den Punkt gebrachte Antrag

Der deutsche Muttersprachler wird daran gewöhnt sein. Sprecher von SVOSprachen dagegen werden diesem lebenslangen Training nie in demselben Maße ausgesetzt sein. V2/ 3 bzw. die relativ ausnahmslose Linksköpfigkeit bei Nomen-Komplement- (statt Nomen-Attribut-)Fügungen wirft ja sofort seinen Selektionsschatten voraus, sodass jedes Element nach dem Prädikat in Zweitstellung ohne Zwischenspeicher abgearbeitet werden kann. ${ }^{11}$

Wie die tiefstrukturierten Attributketten lassen sich auch die charakteristisch langen Wortkompositionen im Deutschen auf der Grundlage von stren-

${ }^{11}$ Es ist unrichtig, dass das Deutsche auch - darin ähnlich, wie behauptet, anderen Sprachen - unterschiedliche Formen mit identischer Funktion und Bedeutung aufweist. Rechtsköpfige Nominalkomposition und linksköpfige Komplementstruktur unterscheiden sich bei gleicher Semantik diskursfunktional nur geringfügig, aber eben doch in einem entscheidenden Merkmal. Man vgl. die folgenden Beispiele (Kapitälchen für Wortakzent):
(i) STRABENbild
$?=$ ?
(ii)
(iv)
Lied der STRABE
(iii) GoTTEsliebe
$?=$ ?
Liebe GOTTES

Wie Relativsätze sind (ii) und (iv) verarbeitungserleichternd, präponierte Attributkomplexe ebenso wie (i) und (iii) dagegen parsingerschwerend. Man beachte, dass der links vom Nominalkopf stehende Attributkomplex infolge der Frühstellung den Status eines Diskursthemas zugewiesen bekommt, während der nachgestellte Relativsatz deshalb thematische Funktion hat, weil er auf eine niedrigere Texthierarchie gestellt ist (Ehlich 1994). 
ger Rechtsköpfigkeit sowie Cinques Fokusnullhypothese (Cinque 1993) systematisch beschreiben und in völligem Einklang mit der Satz- und Attributstruktur erklären. Dabei sind Thema/Backgrounding und Fokus/Rhema/ Foregrounding eindeutig syntaktische Identifikate. In dieser kategorienübergreifenden globalen Erklärbarkeit liegt die besondere, von keiner anderen germanischen Sprache geteilte Eigenschaft des Deutschen; erst diese begründet die strukturglobale Diskursprominenz des Deutschen. Ich wiederhole, was diese besonderen Eigenschaften des /Deutschen sind: (i) die Einfachheit, mit der im syntaktischen Mittelfeld die nominalen Satzglieder stellungs-, fokus- und damit diskursmanipulierbar werden; und (ii) strenge Rechtsköpfigkeit, somit der Modifikatoren- und Komplementaufbau nach links, der global für die 3 Großkategorien - Satz, DP/NP und Attributkomplex sowie mehrfache Nominalkomposition gilt. Man beachte, dass diese kategorienübergreifende Einheitlichkeit in den germanischen SVO-Sprachen gebrochen ist: die Komplementstruktur bei SVO ist linksköpfig/rechtsläufig, die Attribut- und die Worstellungsstruktur dagegen sind rechtsköpfig/ linksläufig. Das erfordert bei diesen 3 Großkategorien wechselnde Parsingstrategien. Im Deutschen dagegen kommt man mit ein und derselben Verarbeitungsstrategie aus. Das ist eine ganz wesentliche Vereinfachung.

\section{Morphologische Redundanz schafft Freiplätze für klare und pragmatikreiche Diskursstruktur: das Deutsche als schwierige L2-Sprache, aber als struktursparsame und diskursintensionsreiche Hörsprache}

Es lässt sich für die beschriebene Balance zwischen morphologischer ,Redundanz' und intensional reicher Diskursstruktur des Deutschen vor allen anderen germanischen Sprachen ein virtuelles Evolutionsszenario unter darwinschen Auslesekriterien entfalten. SVO ist, wie wir gesehen haben, eine auf schnelles Verständnis ausgerichtete Wettbewerbsstrategie, die sich dort bewähren wird (bewährt hat), wo die Grammatik unter Polyglottieverhältnissen schnell durchschaut werden musste. Dies traf in jedem Fall auf das Englische zu und was, wie ebenfalls mit Nachdruck durch McWhorter (2001) festgestellt wurde, in den frühen skandinavischen Sprachen vorgelegen haben muss. Hier sind verschiedene sozial-demoskopische Szenarien in der Geschichte der germanischen Stämme vorstellbar, die hier im einzelnen nicht weiter verfolgt werden. Ein solches Szenario ist wohl der Regelfall in der historischen Verkehrsdiaspora in großen, ja selbst mittelgroßen geopolitischen Arealen. D. h. asymmetrisches V2 mit unmittelbarer Kongruenz- und Valenzmarkierung ist angesichts einer solchen verkehrsintensiven Situation der natürliche und erwartbare Sprachtypus. Es ist bedeutsam zu sehen, dass sich genau dies entfaltete, sobald sich die römischen Kolonien in Mitteleuropa vom Lateinischen emanzipiert hatten und ihre eigenen sprachlichen romanischen Ableger ausbauten: dabei galt das Lateinische mit Vletzt (oder 
V1, mit eigener zentripetaler Binnenstruktur) nach wie vor als Schreibsprache, von der sich die Sprechsprachen eigenständig und zwar nach Sprechsprachkriterien abhoben. Ähnliches galt fürs Englische in seiner Mischsiedlungsgeschichte ebenso wie in der Festlandskandinavia. Die festland-westgermanischen Stämme dagegen waren nie in einer solchen Abhängigkeit von einer einzigen Mutter-Sprache. Ein konservierender eigensprachlicher Schriftkode spielte hier über Jahrhunderte keine Rolle. Was sich letztlich zu den deutschen Stämmen verband, war areal enger beisammen und stand sich sprachlich weitgehend sehr nahe. Hier lag das SVO fordernde und fördernde Polyglottie- und Schnellüberbrückungsszenario nicht vor. Vielmehr konnte man sich auf die die politische und kulturelle Sozialisierung sichernde Diskursfunktion der gemeinsamen Sprache konzentrieren.

Ich habe diese Vorstellung unter ,evolutionären' Vorzeichen entwickelt, aber mittels einer Spätzeit, als bereits nahezu alles in den germanischen Sprachen geregelt sein musste, illustriert. Man mag mir zugestehen, dass dies einen spekulativen Vergleich mit weiter unbekannten prähistorischen Szenarien erlaubt. Es scheint mir gleichwohl, dass ein solcher Vergleich nicht ohne Erklärwert ist. Sprachen wie das Deutsche, die ihre Diskursprominenz durch SVOV und daraus folgend durch ein weites Mittelfeld mit extensiven Scramblingmöglichkeiten sichern, zeichnen sich dadurch aus, dass sie die Balance zwischen reicher Morphologie - und damit schwererer $\mathrm{Zu}$ gänglichkeit durch Anderssprechende - und intensionsreichem Diskursfluss - und damit hohem Grad an Gleichlauf zwischen Gemeintem und Gesagtem - zugunsten einer Verdichtung sozialsprachlicher Beziehungen treffen.

Dass wie in modernen Zeiten dann Unbehagen dort entsteht, wo Sprechern solcher diskursprominenter Sprachen jene Sprache als lingua franca abverlangt wird, die strukturell solchen flüssigen Textverdichtungen in weit geringerem Maße entspricht, nämlich das Englische, und wo weiter beim deutschen Englischsprecher dauernd Ungenügen über die relativ sperrigen Vertextungsmittel des Englischen aufkommt einerseits und andererseits über die Vorwürfe, er, der Deutsche, schreibe/spreche ein zu kompliziertes, zu lange Sätze bauendes Englisch - dieses Unbehagen auf deutschsprachiger Seite ist somit strukturell-typologisch vorprogrammiert. Man erinnere sich: der Deutsche ist gewohnt sein Zwischenspeichergedächtnis in weit aktiverem Maße zu trainieren und zu strapazieren. Er könnte, so ließe sich beinahe sagen, aus dem Englischen textverdichtend und pragmatikeinbeziehend (illokutive Modalpartikel!) viel mehr machen, wenn die Struktur des Englischen dies nur zuließe. Aber natürlich, sie lässt dies nicht zu (weil der englische Satz kein Mittelfeld hat und damit weder Scrambling noch Modalpartikel zulässt). Das ist, auf den linguistisch-typologischen Punkt gebracht, das Dilemma des heutigen deutschen Englischsprechers - dem gar nichts anderes übrig bleibt als die Flucht nach vorne anzutreten, noch besser Englisch zu lernen und darauf zu setzen, dass das Deutsche doch wieder einmal Welt- und Wissenschaftssprache wird. Die Zeichen stehen diesbezüglich freilich nicht gut. 
Unter dieser Sicht lassen sich auch konkretere Positionen hinsichtlich des evolutionären Begriffs der Exaptation beziehen - eines Begriffs, den Forscher wie Lass (1990) und McWhorter (2001) mit Blick auf das in hohem Maße formenabbauende Englisch gebraucht haben. ,Exaptation' ist, wir erinnern uns, das Gegenteil von (darwinianisch zu verstehender) Adaptation: der uneigentliche Funktionseinsatz einer Form, die ihre ursprüngliche sprachliche Funktion infolge formaler Erosion verloren hat (vgl. Haspelmath 2000).

(i) Was die Reflexivverwendung als Diathesenanzeige anlangt, geht die Klassifikation , arbiträre Exaptation des Pronomens für Verbgenuszwecke" sicher an der Einsicht vorbei, dass Reflexivum und Passiv ganz ähnliche Funktionen haben - d.h. dass das Reflexiv ebenso wie Passivierung detransitivierend und deagentivierend wirken. Das sind tiefe semantisch-syntaktische Zusammenhänge, die das Deutsche mit der Reflexivdiathese (,Dekausativ') tief ausschöpft - anders als das Englische, das Niederländische und das Friesische.

(ii) Sicherlich liegt nicht arbiträre Exaptation mit der dreifachen Genusklassifikation und Genusanzeige am Artikelwort und dem attributiven Adjektiv vor: es liegt sehr wohl Exaptation vor, aber eben zu höherem Zweck, nämlich zur Diskursstrukturierung.

(iii) Ebenso wenig lässt sich arbiträre Exaptation für das indefinite Pronomen man geltend machen dann, wenn man bedenkt, dass das spezifische indefinite Pronomen generale die Kontextabhängigkeit deutlich verringern hilft, das Online-Verständnis des Deutschen linear fortschreitend abschätzbar macht, jedenfalls im Vergleich zu jenen Sprachen, die Homonymien wie andere germanische Sprachen und insbesondere das Englische auftreten lassen. Im Wesen unterstreicht dies unsere These von der hohen Diskursprominenz des Deutschen, seiner kommunikativen Verlässlichkeit und der Tendenz zu Eindeutigkeit.

(iv) Schließlich erleichtern die hohen formalen Paradigmenzahlen die Klassenbildung nach morphosyntaktischer Selektion und damit, wie wir mehrfach argumentiert haben, vor allem über die Mittelfeldstrecke bis zum lexikalischen Verb in Letztstellung die Interpretationsarbeit in den linearen Verarbeitungsschritten des Satzes. Das Englische als Gegenpol zur Verarbeitungsstrategie des Deutschen braucht derartige Klassenbildungen nicht, da das Verb in Frühstellung (V2/3) in jedem Falle Valenzund Rektionsbedingungen vorwegnimmt.

Wir haben gesehen, dass der Nachteil, dass das Deutsche auf strenge Frühstellung (wie ne. V2) des finiten Prädikats verzichtet, dadurch aufgewogen wird, dass Diskursfunktionen in der verschiebungsfreundlichen (scrambling) Periode des Mittelfelds ohne weiteren Ableitungsaufwand signalisiert werden können. Dies vor allem ist die Balance, die zwischen einer formsparsamen, aber versetzungssperrigen Sprache wie dem Englischen einerseits und 
dem formaufwendigen, aber versetzungsfreudigen Deutschen herzustellen ist. Keine der beiden Strategien hat a priori eindeutige Vorteile oder Nachteile, es sei denn man macht die Rechnung des Erlernens als Zweitsprache auf. Da scheint das Deutsche im Nachteil zu sein. Aber das kann ja kein Kriterium in der Sprachevolution gewesen sein. Vielmehr muss sich das Deutsche - skizzieren wir nun einmal eine evolutionäre Situation - als textdichtere, Sprecherintention und Sprechermutmaßungen über Diskursbeteiligte und Kotexte feinkörniger, also intensional reicher wiedergebende Intralingua, also in seiner sozial-effizienten Funktion innerhalb einer kompetenten Sprechergemeinschaft besonders bewährt haben. Das Englische dagegen, so könnte man sagen, schöpft seine Kraft aus der Tatsache, dass es die schnelle sprachliche und damit soziale Integration Fremdsprachiger besser fördert. Aber das spiegelt ja nun gerade die historische Situation der britischen Inseln und ihrer Besiedlungswellen ebenso wie die Situation heute, in der sich das Englische ohne sozial-politischen Druck als die am einfachsten zugängliche Lösung einer Interlingua herausgebildet hat.

\section{Schluss und Folgerung}

Ziel der Überlegungen war zu zeigen, dass man zum einen davon sprechen kann, dass das Deutsche aus dem Blickwinkel der übrigen germanischen Sprachen überspezifizierende Merkmale seines morphologischen und syntaktischen Bestandes erhalten hat, während die Verwandten diese Überspezifikation in unterschiedlichem Grade abgebaut haben, das Englische dabei am radikalsten. Dass dabei nicht nur Flexionsabbau bzw. eine Tendenz zur Analytizität die treibende Kraft gewesen sein kann, haben die Einsichten in Funktion und Wirkung von semantisch-syntaktischer Klassenbildung (Selektion) gezeigt. McWhorter geht so weit, dem Abbau der ursprünglichen Überspezifikation - dem Abbau einer Balance zwischen formaler Redundanz und dem Reichtum an diskursfördernden Mitteln - typologische Kausalität zuzusprechen (vgl. in ähnlichem Sinne Lass 1987, S. 317-32). Die hier vorgelegte Determinante lautet anders. Es scheint, dass Sprachen in einer kritischen Evolutionsphase die Entscheidung zwischen optimaler grammatischer Durchsichtigkeit, die zu SVO führen kann, und Diskursoptimierung treffen. Letztere ist mit SVO nicht verträglich, sondern benötigt ein strukturell offenes Mittelfeld, das nur durch die Verbklammer gewährleistet ist. Alles andere - Morphologieredundanz (die natürlich unter unserem Argumentationswinkel nicht redundant ist!), hohe lexikalisch-grammatische Intensionalität, weitgehend Parallelen zwischen Satzsemantik und Syntax (Skopus) - folgt daraus. Es muss in der historischen Ausbildung des Deutschen demopolitisch kritische Perioden gegeben haben, zu denen diese Entscheidungen PRO SVOV+ MORPHOLOGIEERHALT und CONTRA MORPHOLOGIEVEREINFACHUNG+Verzicht AUF DiskuRSOPTIMIERUNG getroffen wurden. Ich habe Andeutungen dazu gemacht, wie man sich das vorstellen kann. 


\section{Literatur}

Abraham, Werner (1987): Burzio trifft Wulfila. Zu den distributionellen Eigenschaften von wairdan, ,werden" und wisan ,sein" im gotischen Passiv. [Groningen Papers in Theoretical and Applied Linguistics - TTT 9]. Groningen.

Abraham, Werner (1989): Futurtypologie in den germanischen Sprachen. In: Abraham, W./Janssen, Th. (Hg.): Tempus-Aspekt-Modus: die lexikalischen und grammatischen Formen in den germanischen Sprache. [Linguistische Arbeiten 237]. S. 345-390. Tübingen: Max Niemeyer.

Abraham, Werner (1992): The emergence of the periphrastic passive in Gothic.In: Leuvense Bijdragen $81 / 1-3$, S. 1-15.

Abraham, Werner (1995): German: „cold and male“ - Dutch: „warm and female“. Reflections on a realistic linguistic typology. In: Shannon; Th./Snapper, J. P. (Hg.): The Berkeley Conference on Dutch Linguistics 1993. Dutch linguistics in a changing Europe. Lanham/New York/London: University Press of America. S. 171-210.

Abraham, Werner (1997a): Kausativierung und Dekausativierung: zu Fragen der verbparadigmatischen Markierung in der Germania. In: Birkmann, Th./Klingenberg, H./Nübling, D./Ronneberger Sibold, E. (Hg.): Vergleichende germanische Philologie und Skandinavistik. Festschrift für Otmar Werner. Tübingen: M. Niemeyer. S. 13-28.

Abraham, Werner (1997b): The base structure of the German clause under discourse functional weight: contentful functional categories vs. derivative functional categories. In: Abraham, W./Gelderen, E.v. (Hg.): German: syntactic problems - problematic syntax. [Linguistische Arbeiten 374]. Tübingen: M. Niemeyer. S. 11-42.

Abraham, Werner (2000): Zwischen extensionaler Ökonomie und intensionaler lokalistischer Präzisierung: Dativobjekte im Deutschen und ihre Wiedergabe im kasuslosen Niederländischen. In: Bittner, A./Bittner, D./Köpcke, K.-M. (Hg.): Angemessene Strukturen: Systemorganisation in Phonologie, Morphologie und Syntax. Hildesheim u. a.: Georg Olms, S. 299-316.

Abraham, Werner (2001): Gibt es im Deutschen eine Klasse von Präpositionen mit Doppelrektion? In: Deutsche Sprache (DS) 29, S. 63-75.

Abraham, Werner/Conradie, Jac (2001): Präteritumschwund und Diskursgrammatik. Amsterdam-Philadelphia: John Benjamins.

Baptista, Marlyse (2000): Verb movement in four creole languages: A comparative analysis. In: McWhorter, John (Hg.): Language change and language contact in pidgins and Creoles S. 1-33. Amsterdam: John Benjamins.

Barnes, William (1886): A glossary of the Dorset dialect with a grammar of its word shapening and wording. London: Trübner \& Co.

Bayer, Josef (Hg.) (1994): Sentence processing and the nature of the human syntactic parser. Sonderheft Folia Linguistics Europaea. Acta Societatis Linguisticae Europaeae XXVIII/1-2.

Bickerton, Derek (1995): Language and human behavior. Seattle: University of Washington Press.

Bobalijk, Jonathan/Thráinnson, Höski (1997): Two heads aren't always better than one. In: Syntax 1, S. 37-71.

Brenner, Oscar (1882): Altnordisches Handbuch. Leipzig: T.O. Weigel.

Cinque, Guglielmo (1993): A null theory of phrasal and compound stress. In: Linguistic Inquiry 24/2, S. 239-298.

Chomsky, Noam (1976): Deep structure, surface structure, and semantic interpretation. In: Studies on semantics in generative grammar. Den Haag: Mouton. S. 62-119.

Clahsen, Harald/Muysken, Pieter (1986): The availability of Universal Grammar to adult 
and child learners: a study of the acquisition of German word order. In: Second Language Research 1, S. 93-119.

Crowley, Terry (2000): Simplicity, complexity, emblematicity and grammatical change. In: Siegel, Jeff (Hg.) (2000): Processes of language contact: studies from Australia and the South Pacific. S. 175-93.

Crystal, David (1987): The Cambridge Encyclopedia of Language. Cambridge: Cambridge University Press.

Curme, George O. (1952): A grammar of the German language. New York: Frederick Ungar. Czepluch, Hartmut (1988): Kasusmorphologie und Kasusrelationen: Überlegungen zur Kasustheorie am Beispiel des Deutschen. In: Linguistische Berichte 116, S. 275-310.

Dalton-Puffer, Christiane (1997): Middle English as a creole and its opposite: on the value of plausible speculation. In: Fisiak (1997) (ed.), S. 35-50.

DeGraff, Michel (Hg.) (1999): Language creation and language change. Cambridge, MA: The MIT Press.

Den Besten, Hans (1983): On the interaction of root transformations and lexical deletive rules. In: Abraham, Werner (Hg.): On the formal syntax of the Westgermania: papers from the Third Groningen Grammar Talks. Groningen, January 1981. Amsterdam: John Benjamins. S. 47-131.

Diesing, M. (1997): Yiddish VP order and the typology of object movement in Germanic. In: NLLT 15, S. 369-427.

Domingue, Nicole Z. (1977): Middle English: another creole? In: Journal of Creole Studies 1, S. 89-100.

Edwards, John. (1994): Multilingualism. London: Penguin.

Ehlich, Konrad (1994): Funktion und Struktur schriftlicher Kommunikation. In: Günther, H./Ludwig, O. et al. (Hg.): Schrift und Schriftlichkeit - Writing and its use. Ein interdisziplinäres Handbuch internationaler Forschung - An interdisciplinary handbook of international research. Berlin: de Gruyter. S. 18-41.

Fabricius-Hansen, Cathrine (2002): Deutsch - eine ,reife' Sprache. Plädoyer für Komplexität. Vortrag IDS-Jahrestagung 12.-14.3.2002. Beispielvorlage.

Fischer, Olga (1992): Syntax. In: The Cambridge history of the English language. Vol. 2, ed. by Norman Blake, S. 207-408. Cambridge: Cambridge University Press.

Fischer, Olga (1997): Infinitive marking in Late Middle English: transitivity and changes in the English system of case. In: Fisiak (1997), S. 109-134.

Fisiak, Jacek (1997): Studies in Middle English linguistics. Berlin: Mouton de Gruyter.

Gil, David (2001): Creoles, complexity, and Riau Indonesian. In: Linguistic Typology.

Haberland, Hartmut (1994): Danish. In: König, Ekkehard/van der Auwera, Johan (Hg.) (1994): The Germanic languages. London: Rotledge. S. 313-348.

Haider, Hubert (1993): Deutsche Syntax - generativ. Tübingen: G. Narr.

Haider, Hubert (1997): Projective economy. In: Abraham, W./Gelderen, E.v. (Hg.): German: syntactic problems - problematic syntax. Tübingen: M. Niemeyer. S. 83-103.

Haider, Hubert/Rosengren, Inger (1998): Scrambling. Lund. (Sprache und Pragmatik 49).

Haiman, John (1983): Iconic and economic motivation. In: Language 59, S. 781-819.

Hamp, Eric (1965): The Albanian dialect of Màndres. In: Die Sprache 11, S. 137-54.

Haspelmath, Martin (1998): How Young is Standard Average European? In: Language Sciences 20, S. 271-87.

Haspelmath, Martin (1999): External possession in a European areal perspective. In: Payne, Doris L./Barshi, Immanuel: External possession. Amsterdam: John Benjamins. S. 109-35.

Haspelmath, Martin (2000): Optimality and diachronic adaptation. In: Zeitschrift für Sprachwissenschaft, S. 1-25. 
Hawkins, John A. (1986): A comparative typology of English and German: unifying the contrasts. Austin-London: University of Texas/Routledge/Croom Helm.

Heine, Bernd (1975): Some generalizations of African-based pidgins. Vortrag International Conference on Pidgins and Creoles, Honolulu.

Heusler, Andreas (1950): Altisländisches Elementarbuch. Heidelberg: Carl Winter.

Höhle, Tilman N. (1982): Explikation für ,normale Betonung‘ und ,normale Wortstellung‘. In: Abraham, W. (Hg.): Satzglieder im Deutschen. Tübingen: G. Narr. (Studien zur deutschen Grammatik 15).

Holmes, Philip/Hinchliffe, Ian (1997): Swedish: an essential grammar. London: Routledge. Kayne, Richard (1994): The antisymmetry of syntax. Cambridge, MA: MIT Press.

Kemmer, Suzanne (1993): The middle voice. Amsterdam: Benjamins.

Klaiman, Mimi H. (1991): Grammatical voice. Cambridge: CUP.

König Ekkehard/Haspelmath, Martin (1998): Les constructions à possesseur externe dans les langues d'Europe. In: Feuillet, Jack (ed.): Actance et valence dans les langues de l'Europe. (Empirical Approaches to Language Typology/EUROTYP, 20-2.) Berlin: Mouton de Gruyter. S. 525-606.

Kouwenberg, Silvia (1994): A grammar of Berbice Dutch Creole. Berlin: Mouton de Gruyter.

Kress, Bruno (1982): Isländische Grammatik. München: Max Hueber.

Kroch, Anthony/Taylor, Ann (1997): Verb movement in old and Middle English: dialect variation and language contact. In: Van Kemenade/Vincent, S. 297-325.

Lass, Roger (1987): The shape of English. London: J. M. Dent \& Sons.

Lass, Roger (1990): How to do things with junk: exaptation in language evolution. In: Journal of Linguistics 26, S. 79-102.

Lass, Roger (1992): Phonology and morphology. In: The Cambridge history of the English language (Vol. 2). ed. by Norman Blake. Cambridge: Cambridge University Press. S. 23-155

Leiss, Elisabeth (1985): Zur Entstehung des neuhochdeutschen analytischen Futurs. In: Sprachwissenschaft 10/3-4: S. 250-273.

Lockwood, W.B. (1955): An introduction to Modern Faroese. Copenhagen: Ejnar Munksgaard.

Lockwood, W.B. (1995): Lehrbuch der modernen jiddischen Sprache. Hamburg: Helmut Buske.

Matthews, William K. (1956): The Livonian element in modern Latvian. In: Festschrift für Max Vasmer zum 70. Geburtstag, hg. von Margarete Woltner/Bräuer, Herbert, S. 307-318. Berlin: Veröffentlichungen der Abteilung für slawische Sprachen und Literaturen des Osteuropa-Instituts an der Freien Universität Berlin.

McWhorter John H. (2001): What happened to English? Ms. UCal at Berkeley.

Mitchell, Bruce (1985): Old English syntax, Vol. 1. Oxford: Clarendon Press.

Mitchell, Bruce/Robinson, Fred.C. (1986): A guide to Old English. Oxford: Blackwell.

Mustanoja, Tauno F. (1960): A Middle English syntax. Vol. 1. Helsinki: Société Néophilologique.

O'Grady, William, Michael Dobrovolsky and Mark Aronoff ( $\left.{ }^{3} 1997\right)$ : Contemporary linguistics: an introduction. New York: St. Martin's Press.

Paul, H. (1880): Prinzipien der Sprachgeschichte. Halle: Niemeyer

Peitsara, Kirsti (1997): The development of reflexive strategies in English. In: Rissanen, Matti/ Kytö, Merja/Heikkonen, Kirsi (1997): Grammaticalization at work. S. 277-370. Berlin: Mouton de Gruyter.

Platzack, Christer/Holmberg, Anders (1989): The role of AGR and finiteness in Germanic VO languages.In: Working Papers in Scandinavian Syntax 43, S. 51-76. 
Ponelis, Fritz (1993): The development of Afrikaans. Frankfurt: Peter Lang.

Propst, Ron (2002): Passive and reflexive in the history of Scandinavian. Dissertation Univ. Groningen.

Rissanen, Matti (1999): Syntax. In: The Cambridge history of the English language, Vol. 1, ed. by Roger Lass, S. 187-331. Cambridge: Cambridge University Press.

Rohrbacher, B. (1999): Morphology-driven syntax. A theory of V-to-I raising and prodrop. Amsterdam-Philadelphia: John Benjamins.

Santorini, Beatrice (1993): Yiddish as SVO or SOV In: Abraham, W./Bayer, J. (1993): Dialektsyntax. [Linguistische Berichte. Sonderheft 5]. Westdeutscher Verlag: Opladen.

Schildt, Jochen (1994): Zur Ausbildung des Satzrahmens in Aussagesätzen der Bibelsprache (1350-1550). In: PBB (-Ost), Halle, S. 174-197.

Strang, Barbara M.H. (1970): A history of English. London: Methuen.

Thomason, Sarah Grey/Kaufman, Terrence (1988): Language contact, creolization, and genetic linguistics. Berkeley: University of California Press.

Tiersma, Pieter M. (1985): Frisian reference grammar. Dordrecht: Foris.

Traugott, Elizabeth C. (1972): A history of English syntax: a transformational approach to the history of English sentence structure. New York: Holt, Rhinehart/Winston.

Trudgill, Peter (1999): Language contact and the function of linguistic gender. In: Posnan Studies in contemporary Linguistics 35, S. 133-52.

Vikner, Sten (1995): Verb movement and expletive subjects in the Germanic languages. Oxford: OUP.

Visser, F. Th. (1963): An historical syntax of the English language, Vol. 1. Leiden: E. J. Brill.

Zwart, C.Jan-Wouter (1997): Morphosyntax of verb movement: a minimalist approach to the syntax of Dutch. Dordrecht: Kluwer. 